\title{
Human hip-ankle coordination emerging from multisensory feedback control
}

\author{
Georg Hettich $^{\mathrm{a}, \mathrm{b}, *}$, Lorenz Assländer ${ }^{\mathrm{a}, \mathrm{b}}$, Albert Gollhofer ${ }^{\mathrm{b}}$, Thomas Mergner ${ }^{\mathrm{a}}$ \\ a Neurological University Clinic, Neurocenter, Breisacher Str. 64, 79106 Freiburg, Germany \\ ${ }^{\mathrm{b}}$ Institute for Sport and Sportscience, University of Freiburg, Schwarzwaldstr. 175, 79117 Freiburg, Germany
}

\begin{abstract}
A B S T R A C T
Human sensorimotor control involves inter-segmental coordination to cope with the complexity of a multi-segment system. The combined activation of hip and ankle muscles during upright stance represents the hip-ankle coordination. This study postulates that the coordination emerges from interactions on the sensory levels in the feedback control. The hypothesis was tested in a model-based approach that compared human experimental data with model simulations. Seven subjects were standing with eyes closed on an anterior-posterior tilting motion platform. Postural responses in terms of angular excursions of trunk and legs with respect to vertical were measured and characterized using spectral analysis. The presented control model consists of separate feedback modules for the hip and ankle joints, which exchange sensory information with each other. The feedback modules utilize sensorderived disturbance estimates rather than 'raw' sensory signals. The comparison of the human data with the simulation data revealed close correspondence, suggesting that the model captures important aspects of the human sensory feedback control. For verification, the model was re-embodied in a humanoid robot that was tested in the human laboratory. The findings show that the hip-ankle coordination can be explained by interactions between the feedback control modules of the hip and ankle joints.
\end{abstract}

* Corresponding author at: Neurological University Clinic, Neurocenter, Breisacher Str. 64, 79106 Freiburg, Germany. Tel.: +49 761 27052280; fax: +4976127053100.

E-mail address: georg.hettich@uniklinik-freiburg.de (G. Hettich). 


\section{Introduction}

The many degrees of freedom of the musculoskeletal apparatus and the action-reaction mechanical interactions between body segments make the human sensorimotor control very complex. It has been suggested that humans cope with this complexity by coordinating movements and muscle activations in terms of synergies (Bernstein, 1967). A variety of aspects in relation to the coordination have been considered. In view of the high redundancy in the motor system, it has been argued that it is much simpler for the brain to perform motor tasks by activating pre-established sets of muscles rather than individual muscles (Lockhart \& Ting, 2007; McCollum, Horak, \& Nashner, 1984). It has also been proposed that a few such 'building blocks' suffice to deal with the actuation of a multi-segment body (d'Avella \& Pai, 2010; Ting, 2007; Tresch, Saltiel, \& Bizzi, 1999). In fact, it has been shown that five activation patterns across the body muscles are enough to describe human walking (Ivanenko, Cappellini, Dominici, Poppele, \& Lacquaniti, 2005). The synergies may be established through learning (e.g., trial-and-error learning; Rugy, Loeb, \& Carroll, 2012) and corresponding look-up tables may be used for coordinating the muscle actuations that are required for balancing of external disturbances, as demonstrated in a miniature humanoid robot (Hauser, Neumann, Ijspeert, \& Maass, 2011).

In addition to the redundancy and simplification aspects, there are biomechanical reasons for muscle and movement coordination. For example, an arm reaching movement evokes an opposed acceleration of the adjoining body segments. In order to counteract this acceleration and transfer the reaction force to the external support (often via the feet to the ground), such movements are accompanied by coordinated movements and corresponding contractions of muscles in trunk and legs (Bouisset \& Do, 2008). Similarly, already an acceleration in a single joint results in accelerations of (and joint torques in) adjacent and more distant joints (Zajac \& Gordon, 1989). These 'coupling' forces result from the action-reaction law of physics and complicate the control of multi-segment systems. Furthermore, a reaching movement may endanger the stability of a balancing body by displacing the projection of the whole body's center of mass (body COM) out of the base of support given by the feet. Humans use changes in body posture to counteract such body COM shifts, commonly known as postural adjustments (PAs).

Human movement coordination involves mechanical aspects, musculoskeletal intrinsic properties such as muscle viscosity and stiffness, and neural coordinating mechanisms, as recently discussed for muscle synergies (Kutch \& Valero-Cuevas, 2012). Multi-segment coordination has previously been studied in human balancing in a variety of behavioral scenarios, where the biomechanics can be simplified to a multi-link inverted pendulum in the sagittal plane (e.g., Alexandrov, Frolov, Horak, Carlson-Kuhta, \& Park, 2005; Vernazza-Martin, Martin, Le Pellec-Muller, Tricon, \& Massion, 2006). These studies considered postural responses in hip, knee and ankle joints. However, the forces produced by the knee joints are mainly oriented vertically, while the forces used for balancing are oriented in the horizontal plane (Nashner \& McCollum, 1985). This notion is in agreement with quantitative estimates of the contribution of the knee joints, which was found to be relatively small (Alexandrov, Frolov, \& Massion, 2001a,Alexandrov; Frolov, \& Massion, 2001b; Horak \& Nashner, 1986). In order to study inter-segmental coordination, many studies reduced the biomechanics of a standing human to a double inverted pendulum (DIP) with focus on hip and ankle joint responses. The coordinated responses of these joints are known to depend on disturbance strength and context. Weak disturbances mainly evoke compensatory movements in the ankle joints ('ankle strategy'; Horak \& Nashner, 1986; McCollum \& Leen, 1989; Nashner \& McCollum, 1985). In situations where the ankle joint torque becomes insufficient for balancing, for example when standing on a narrow beam or when the disturbance is very strong, humans use hip joint accelerations that produce shear forces under the feet to counteract body COM excursions ('hip strategy'; Horak \& Nashner, 1986; McCollum \& Leen, 1989; Nashner \& McCollum, 1985). In most situations postural responses involve both, ankle and hip joints (Creath, Kiemel, Horak, Peterka, \& Jeka, 2005; Kiemel, Elahi, \& Jeka, 2008; Park, Horak, \& Kuo, 2004; Runge, Shupert, Horak, \& Zajac, 1999).

During moderate disturbances, another aspect of hip-ankle coordination needs to be considered. In such situations, the primary task is to maintain equilibrium of the whole body, which is mainly 
performed by the ankle joints (Maurer, Mergner, \& Peterka, 2006; Mergner, Maurer, \& Peterka, 2003; Peterka, 2002). The hip joints tend to perform a secondary task, consisting of the stabilization of the vertical orientation of trunk and head and thereby stabilizing the workspaces of the hands and for the eyes (Horak \& MacPherson, 1996; compare Fig. 1A). Minimizing the head movements during body oscillations, called the 'head stabilization in space strategy', is thought to improve sensory feedback from the vestibular and visual cues during dynamic balancing (Assaiante \& Amblard, 1995; Bronstein, 1988; Pozzo, Berthoz, Lefort, \& Vitte, 1990). The head then appears to serve as an egocentric reference for walking and other voluntary activities (Amblard, Assaiante, Fabre, Mouchnino, \& Massion, 1997; Berthoz \& Pozzo, 1988; Grossman, Leigh, Abel, Lanska, \& Thurston, 1988).

Model-based interpretations are helpful to understand the complicated mechanisms underlying human movement control. For example, models using single inverted pendulum (SIP) biomechanics helped to distinguish between neural control mechanisms and biomechanical effects in human balancing (Maurer et al., 2006; Pai \& Patton, 1997; Peterka, 2002; Peterka, 2003; Winter, Patla, Prince, Ishac, \& Gielo-Perczak, 1998). Only few studies used model-based approaches to investigate hip-ankle coordination. Boonstra, Schouten, and van der Kooij (2013) presented a method where two external disturbances were used to identify the contribution of the hip and ankle joints to the balancing. To describe experimental findings, Alexandrov et al. (2005) introduced an eigen-synergy concept where

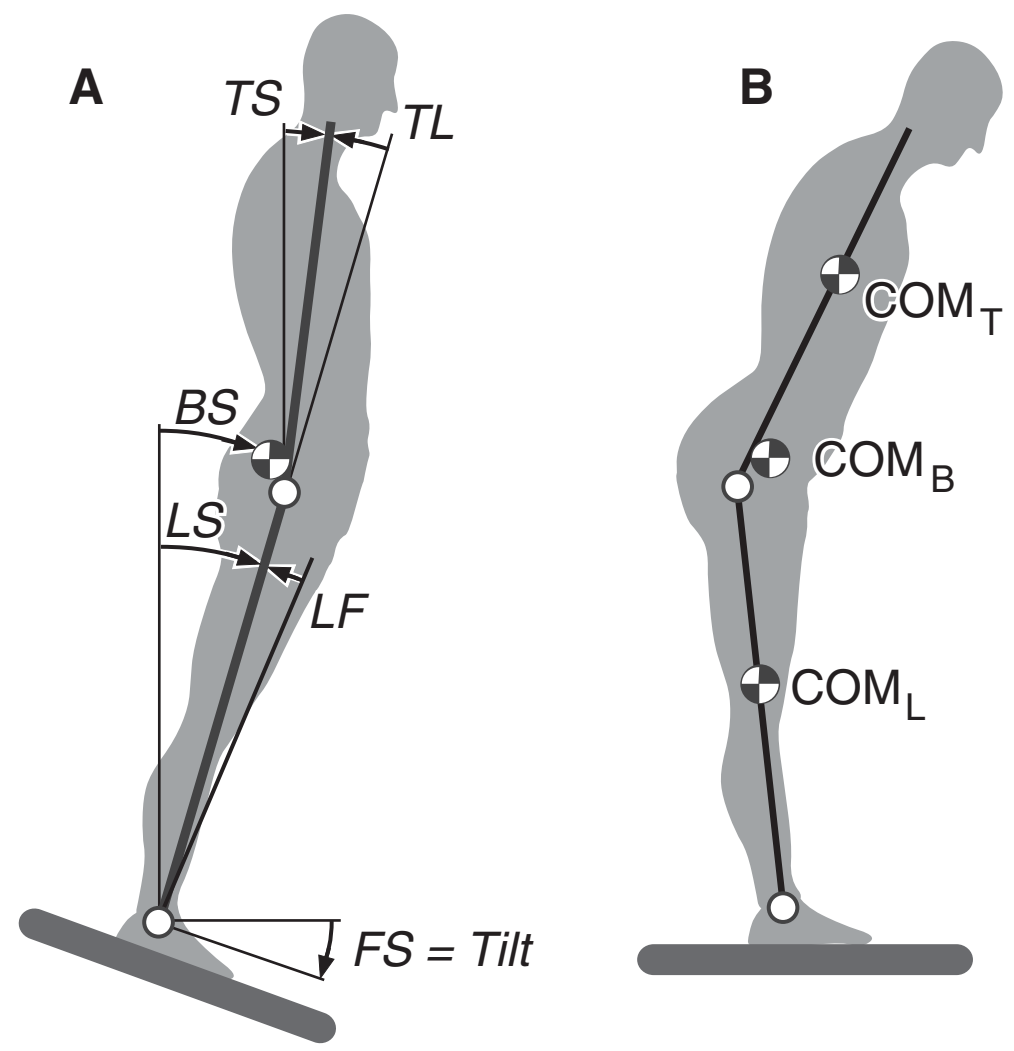

Fig. 1. (A) Conventions of the human biomechanical variables used in this study for double inverted pendulum (DIP) simplification. The upper segment (trunk) comprises head, arms, trunk and the lower segment the legs without the feet. Angles with respect to earth vertical $\left(0^{\circ}\right)$ are the trunk-space angle $T S$ and the leg-space angle $L S$. The foot-space angle $F S$ equals support surface tilt (Tilt) and is referenced to earth horizontal $\left(0^{\circ}\right)$. Hip joint angle is defined as trunk-leg angle $T L$ and ankle angle as leg-foot angle $L F$. (B) Position of whole body center of mass $\left(\mathrm{COM}_{\mathrm{B}}\right) \cdot \mathrm{COM}_{\mathrm{T}}$ and $\mathrm{COM}_{\mathrm{L}}$ refer to the COM of the trunk segment and the leg segment, respectively. 
neural controllers are thought to activate all involved joints such that inter-segmental coupling torques are minimized. As a consequence, the control of each eigen-synergy can work independently and the number of required parameters in the feedback control is reduced as compared to a similar control concept that uses full-state feedback (Park et al., 2004). In these studies the coordination occurs essentially at the level of the controller.

The above-described studies on human movement coordination are mostly dealing with motor and feed forward aspects. In contrast, the role of sensory feedback has received little attention, although sensory feedback is a major constituent of motor control. The importance of sensory feedback is witnessed in neurological patients with impairment at the sensory levels. In such patients feedback mechanisms are disturbed, resulting in ataxia, which includes the loss of the ability to perform smooth movements (Bastian, 1997; Fregly, 1974). The role of sensory feedback and the interaction of sensory cues in human movement coordination are still not fully understood.

Basic and clinical research has shown that human balancing involves joint proprioception, tactile and pressure cues, the vestibular system and vision (Horak \& Macpherson, 1996). The integration of these sensory cues allows humans to adapt their control to changes in environmental conditions and availability of sensory information. This includes the ability to modify the extent to which each sensory cue contributes to the overall torque production (called 'sensory re-weighting'; Maurer et al., 2006; Mergner et al., 2003; Nashner \& Berthoz, 1978; Peterka, 2002; van der Kooij \& Peterka, 2011). Postural control models that investigated sensory integration in anterior-posterior (AP) balancing with more than one degree of freedom (DoF) applied engineering-inspired concepts of sensory integration (Kuo, 2005; van der Kooij, Jacobs, Koopman, \& Grootenboer, 1999). In these models, multiple sensory signals are combined with centrally generated predictions of sensory information in a 'sensory integration center'. The approach uses a Kalman filter to find the most accurate sensory representation for a given environmental situation under noise optimization principles.

A more human-inspired model of sensory integration with automatic sensory re-weighting has been proposed by Mergner, Maurer and Peterka (Maurer et al., 2006; Mergner et al., 2003). The proposed sensory integration mechanisms have been used in a feedback control model in the form of disturbance estimations and compensations (DEC concept; overview, Mergner, 2010). The DEC concept has its origin in psychophysical findings of the integration of proprioceptive and vestibular signals in human self-motion perception (Mergner, Huber, \& Becker, 1997; Mergner, Nardi, Becker, \& Deecke, 1983; Mergner, Siebold, Schweigart, \& Becker, 1991). Based on these experiments, it is assumed that the central nervous system combines sensory information from different sources such that the kinematics and kinetics of a given body segment are known, even though this segment does not directly contain a corresponding sensor. It has been shown in psychophysical experiments that, for example, subjects use vestibular information arising in the head to perceive the kinematic state of the trunk, legs, or foot support surface (Mergner et al., 1997). Also neurophysiological studies suggest that the central nervous system uses processed sensory information when dealing with motion or orientation of body segments (Bosco \& Poppele, 1997; Casabona, Valle, Bosco, \& Perciavalle, 2004). According to these studies, sensory information is exchanged between the controls of the body segments and the corresponding neural networks provide the basis for coordinating movements. The DEC concept has successfully been used to describe human sway behavior in a variety of experimental conditions. However, it has only been established so far for SIP biomechanics. It remains to be shown to which extent it can be extended to describe also the sensory integration in the hip joint control and the hip-ankle coordination of human balancing during perturbed stance.

The current study investigated human balancing during support surface tilts by recording sway responses of the trunk and the leg segments. Model simulations were used to interpret the experimental findings. The proposed model is based on the DEC concept for SIP biomechanics, which was extended to cope with DIP biomechanics.

As computer model simulations may be somewhat unrealistic with respect to sensor inaccuracies and noise, mechanical dead zones, etc., the comparison was extended in two ways. One way was to apply a sensitivity analysis to the model, which tests its control stability across variations of the control parameters. The other way was to implement the control model in a humanoid robot and compare the responses using the same experimental test bed as for human subjects. 
The model was able to resemble the human sway responses when implementing the DEC concept in a modular form and adding an interaction of hip and ankle controls at the sensory level. The approach implied the assumption of a 'modular control architecture' with sensory interactions between the modules, which largely reduces the complexity when controlling several DoF. Preliminary reports on a modular version of the DEC concept with the main focus on coupling torques and sensor fusions have previously been published (Hettich, Fennell, \& Mergner, 2011; Hettich, Lippi, \& Mergner, 2013).

\section{Methods}

The approach used in this study includes two steps. First, human sway responses of the trunk and the leg segment to a broad frequency band support surface tilt stimulus at different amplitudes are measured (Peterka, 2002). This experimental approach has been referred to as 'indirect approach' by van der Kooij, van Asseldonk, and van der Helm (2005), who pointed out that with this method the experimental results reflect both, the biomechanics of the human body (plant) and the stabilizing mechanisms (control). With prior knowledge of the plant, knowledge of the control can be retrieved. In the current study, sway responses of the trunk and the leg segments to the tilt stimuli are characterized in terms of frequency response functions that describe the system's responses to a particular stimulus. The second step applies model simulations (including the plant dynamics) to compare the experimental results with the results obtained from simulations of the hypothesized control model. To test the hypothesized control model additionally in a real world environment, robot experiments are performed where the plant dynamics are authentic and need not be simulated.

\subsection{Subjects}

The experiments were performed in seven healthy subjects (3 female, 4 male; mean age, $28 \pm 3$ years) who were newly recruited compared to our preliminary report (Hettich et al., 2011). Subjects gave their informed consent to the study that was approved by the Ethics Committee of the Freiburg University Clinics.

\subsection{Experimental setup and stimuli}

The experiments were performed using a custom-build 6 DoF motion platform that was actuated by six servomotors (Stuart principle; compare Mergner et al., 2003). Subjects were freely standing on the platform with the heels approximately $10 \mathrm{~cm}$ apart, holding safety ropes, which were loosely hanging from the ceiling, and which provided no spatial orientation cues. Subjects wore headphones and listened to an audio book. This minimized auditory spatial orientation cues and distracted the subjects from the balancing task. The platform was used to present the subjects with AP support surface tilts with the rotation axis approximately through the ankle joints. A $60.5 \mathrm{~s}$ long pseudo-random ternary sequence was used as tilt stimulus (PRTS; Peterka, 2002). It allowed the analysis of the sway responses across a frequency range of $0.017-2.479 \mathrm{~Hz}$.

\subsection{Procedures}

Each experimental session consisted of five experimental trials, where each trial consisted of a repetition of six consecutive $60.5 \mathrm{~s}$ PRTS cycles. Before each trial, the subjects were instructed to close their eyes and to stand comfortably upright. The first trial at $4^{\circ}$ peak-peak (pp) stimulus amplitude was used to accustom subjects with the experimental setup and was not included in the analysis. Succeeding, four trials with pp stimulus amplitudes of $1^{\circ}, 2^{\circ}, 4^{\circ}$, and $8^{\circ}$ (Fig. 2, first column) were presented in randomized order. Additional visual conditions were tested in the same experiment but not included in this paper. Subjects were given breaks of 1-3 min between trials. Each subject performed the experiment twice, with either one hour break between sessions or with the two sessions on different days. Conventions of the human biomechanical variables used in this paper are given in Fig. 1A. 


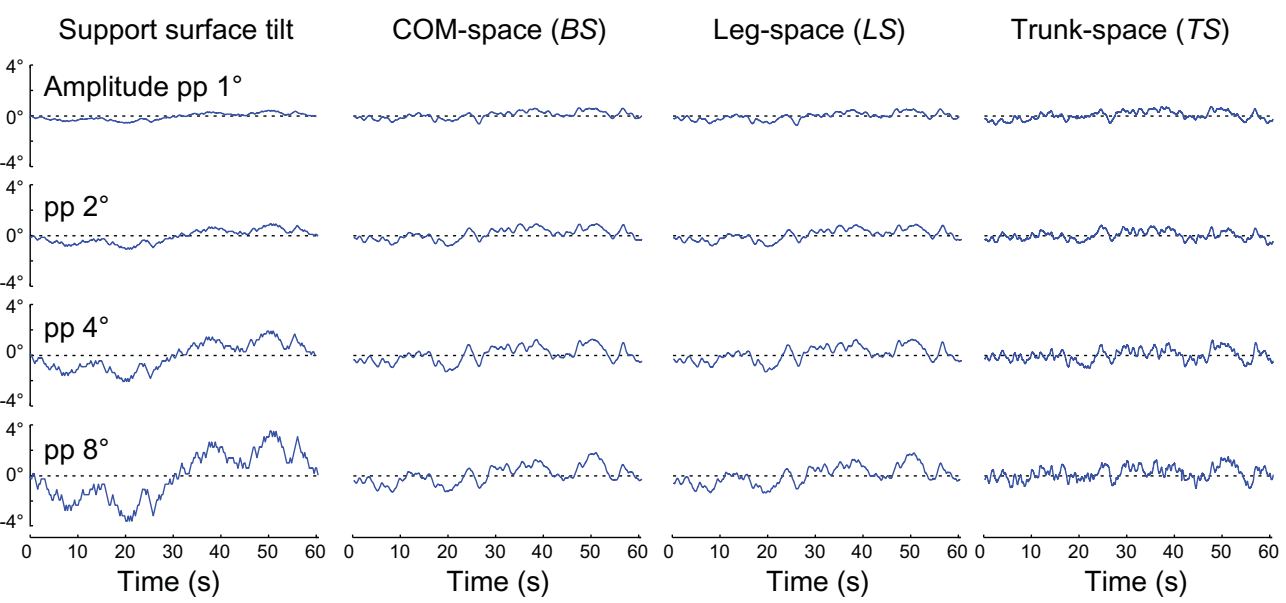

Fig. 2. Representative sway responses of one subject to different support surface tilt stimuli. Columns: tilt stimulus sequence and angular excursions of body COM-space $(B S)$, leg-space $(L S)$, and trunk-space (TS). Rows: four different peak-peak tilt amplitudes ( $\mathrm{pp} 1^{\circ}, 2^{\circ}, 4^{\circ}$, and $8^{\circ}$ ). Each response represents the averages of $2 \times 5$ pseudo-random ternary sequence (PRTS) stimulus cycles.

\subsection{Data acquisition}

AP displacements of hip and shoulder were measured using an opto-electronic device with active body markers (Optotrak 3020; Waterloo, Canada). AP center of pressure (COP) shifts under the feet were recorded using a force-transducing platform (Kistler, platform type 9286, Winterthur, Switzerland). Tilt stimulus, marker displacements, and COP shifts were recorded via an analog/digital converter on a PC with a sampling rate of $100 \mathrm{~Hz}$ using custom-made software programmed in LabView (National Instruments, Austin, USA).

\subsection{Data analyses}

Data processing was performed using custom-made software programmed in Matlab (The MathWorks, Natick, USA). The angular excursions of the leg and trunk segments with respect to the earth vertical (leg-space angle, $L S$, and trunk-space angle, $T S$ ) were calculated from horizontal displacements and the manually measured heights of the markers using trigonometric functions. Subjects' anthropometrics were calculated according to Winter (1990) and are given in Table 1. Using hip and shoulder displacements, angular excursion of the body COM with respect to the vertical (body-space angle; $B S$ ) was calculated thereof (Fig. 1A).

For each individual PRTS cycle the power spectra of stimulus and response as well as the corresponding cross power spectrum were calculated using a discrete Fourier transform. The spectra were calculated over the tilt frequency range of $0.017-2.479 \mathrm{~Hz}$, smoothing the values for high tilt frequencies by averaging across adjacent spectral points in the complex domain (Peterka, 2002). Frequency response functions (FRFs) were calculated by dividing the cross power spectra by the power spectra of the stimulus. The FRFs from support surface tilt (stimulus) to BS (response) characterized whole body balancing at the ankle joint, henceforth called Tilt-to-BS FRFs. Sway responses of the leg and trunk segments to support surface tilt are calculated in analogous form and expressed as Tilt-to-LS FRFs and Tilt-to-TS FRFs, respectively.

The first cycle of each trial was discarded to avoid transient effects. The FRFs calculated from the remaining cycles were averaged across the remaining 70 cycles ( 7 subjects; 2 trials; 5 cycles) and gain and phase over frequency were calculated thereof. Gain, calculated using the absolute value of the FRF at each frequency, resembles the amplitude ratio between sway response and tilt stimulus. A gain of 
Table 1

Anthropometric and control parameters used in the model simulations (switch setting $l s^{\prime}$ ). Parameters obtained in the control experiment are shown in parentheses.

\begin{tabular}{|c|c|}
\hline \multicolumn{2}{|l|}{ Anthropometrics } \\
\hline Body mass (excluding feet) $m_{B}[\mathrm{~kg}]$ & 66.99 \\
\hline Leg segment mass (both legs) $m_{L}[\mathrm{~kg}]$ & 20.21 \\
\hline Trunk mass $m_{T}[\mathrm{~kg}]$ & 46.78 \\
\hline Body height (excluding feet) $l_{B}[\mathrm{~m}]$ & 1.67 \\
\hline Leg length $l_{L}[\mathrm{~m}]$ & 0.85 \\
\hline Body COM height (above ankle joint) $h_{B}[\mathrm{~m}]$ & 0.96 \\
\hline Leg COM height $h_{L}[\mathrm{~m}]$ & 0.47 \\
\hline Trunk COM height $h_{T}[\mathrm{~m}]$ & 0.31 \\
\hline \multicolumn{2}{|l|}{ Ankle Module } \\
\hline Proportional part of ankle controller [ $\left.\mathrm{Nm} \mathrm{rad}^{-1}\right]$ & 629.24 \\
\hline Derivative part of ankle controller [ $\mathrm{Nm} \mathrm{s}^{-1} \mathrm{rad}^{-1}$ ] & 188.77 \\
\hline Passive ankle stiffness [ $\left.\mathrm{Nm} \mathrm{rad}^{-1}\right]$ & 94.39 \\
\hline Passive ankle viscosity [ $\mathrm{Nm} \mathrm{s}^{-1} \mathrm{rad}^{-1}$ ] & 28.31 \\
\hline Lumped time delay $\Delta t_{A}[\mathrm{~s}]$ & 0.18 \\
\hline Gain factor of If proprioceptive signal & 0.85 \\
\hline Gain factor in $T_{A_{-} \text {grav }}$ estimate & 0.54 \\
\hline Threshold in $T_{A \_g r a v}$ estimate [rad] & 0.0028 \\
\hline Time constant of low pass filter in $T_{A_{-} \text {grav }}$ estimate [s] & 15.00 \\
\hline Gain factor of low pass filter in $T_{A_{-} \text {grav }}$ estimate & 0.16 \\
\hline Gain factor in $F S$ estimate & 0.72 \\
\hline Threshold in FS estimate $\left[\mathrm{rad} \mathrm{s}^{-1}\right]$ & 0.0064 \\
\hline \multicolumn{2}{|l|}{ Hip Module } \\
\hline Proportional part of hip controller [ $\mathrm{Nm} \mathrm{rad}^{-1}$ ] & 143.97 \\
\hline Derivative part of hip controller [ $\mathrm{Nm} \mathrm{s}^{-1} \mathrm{rad}^{-1}$ ] & 28.79 \\
\hline Passive hip stiffness $\left[\mathrm{Nm} \mathrm{rad}^{-1}\right]$ & 21.59 \\
\hline Passive hip viscosity $\left[\mathrm{Nm} \mathrm{s}^{-1} \mathrm{rad}^{-1}\right]$ & 4.32 \\
\hline Lumped time delay $\Delta t_{H}[\mathrm{~s}]$ & 0.07 \\
\hline Gain factor of $t l$ proprioceptive signal & 0.85 \\
\hline Gain factor in $T_{H_{\text {grav }}}$ estimate & $0.95(0.45)$ \\
\hline Threshold in $T_{H \_ \text {grav }}$ estimate [rad] & $0.0018(0.0016)$ \\
\hline Time constant of low pass filter in $T_{H_{\text {_grav }}}$ estimate [s] & 15.00 \\
\hline Gain factor of low pass filter in $T_{H_{\text {_grav }}}$ estimate & $0.0045(0.11)$ \\
\hline Gain factor in $L S$ estimate & $0.69(0.57)$ \\
\hline Threshold in $L S$ estimate $\left[\mathrm{rad} \mathrm{s}^{-1}\right]$ & $0.0017(0.0063)$ \\
\hline
\end{tabular}

unity indicates that the response amplitude equals the stimulus amplitude at the given frequency, while a gain of zero indicates that the stimulus does not evoke any correlated sway response (i.e., the stimulus is ideally compensated). The phase curves, calculated by the inverse tangent of the real and imaginary part of the FRF, resemble the corresponding temporal relation between stimulus and response. Coherence function estimates were calculated by dividing the squared absolute values of the cross power spectrum by the product of the stimulus and response power spectra at each frequency. Coherence function values are a measure for the frequency dependent signal to noise ratio of the system. 95\% confidence limits were calculated for gain, phase and coherence functions based on F-statistics (Otnes \& Enochson, 1972).

The Tilt-to-TS FRFs characterize the trunk responses to support surface tilt, which includes compensation mechanisms of the ankle and the hip joint. To characterize the relationship between trunk and leg movement, the ratio of the gain values and the difference of the phase values between the Tilt-toTS and Tilt-to-LS FRFs were calculated (Kiemel et al., 2008).

\subsection{Control experiment}

The assumption of a modular control architecture, where the hip joint control replicates the DEC control of the ankle joint, was tested with two of the subjects in a control experiment. In this experiment, the legs of the subjects were fixed to the motion platform using a mechanical setup and the 
platform was rotated about an axis passing through the subjects' hip joints. The same PRTS stimulus sequence as in the free-standing experiments with tilt amplitudes of $\mathrm{pp} 1^{\circ}, 2^{\circ}$, and $4^{\circ}$ was used. In this setup, the hip joint stimuli consisted of a PRTS rotation of the leg segment around the hip joint and is thereby comparable to the support surface tilt stimuli applied to the ankle joint in the free-standing setup. FRFs from the leg rotation stimuli to the trunk responses ( $L S_{S T I M}$-to-TS FRFs) were calculated as described before for the free-standing experiment and averaged across the two subjects.

\section{Results}

Fig. 2 shows $B S, L S$, and $T S$ responses to support surface tilt from one representative subject for the four tilt stimulus amplitudes ( $\mathrm{pp} 1^{\circ}, 2^{\circ}, 4^{\circ}$, and $8^{\circ}$ ). Considering first the $B S$ responses, i.e., the tilt evoked body COM excursions around the ankle joints, $B S$ amplitude was found to increase with increasing tilt amplitude. However, the increase in $B S$ amplitude was not proportional to that of the tilt amplitude, but clearly smaller. This behavior is known as amplitude non-linearity (Maurer et al., 2006; Peterka, 2002). The $L S$ responses in Fig. 2 closely resemble the $B S$ responses. This reflects the fact that hip joint and body COM are located close to each other (compare Fig. 1B). Finally, also the TS responses were roughly similar to the $B S$ responses, apart from additional high-frequency components.

Averaged FRFs from support surface tilt stimuli to the $B S$ responses (Tilt-to-BS FRFs) across all subjects for the four peak-peak tilt amplitudes are plotted in Fig. 3Aa in terms of gain and phase together with the corresponding coherence curves. The gain curves reflect the aforementioned amplitude nonlinearity. At the lowest tilt frequency $(0.017 \mathrm{~Hz})$, gain values decreased from approximately unity gain for the pp $1^{\circ}$ stimulus amplitude to a gain of about 0.5 for the $\mathrm{pp} 8^{\circ}$ stimulus amplitude. At higher tilt frequencies up to approximately $0.3 \mathrm{~Hz}$, the gain curves increase and diverge. Gain plateaus are reached between $0.4-0.5 \mathrm{~Hz}$, while at higher frequencies $(0.6-2.2 \mathrm{~Hz})$ the $B S$ gain curves decrease and converge. The phase curves of $B S$ in Fig. 3Aa almost coincide with each other, being essentially in phase with the tilt at $0.2 \mathrm{~Hz}$. At frequencies below $0.2 \mathrm{~Hz}$, they show a small phase lead. At frequencies above $0.2 \mathrm{~Hz}$, the phase curves slightly diverge and develop a lag, which reaches at the highest frequency $-181^{\circ}$ for the $\mathrm{pp} 8^{\circ}$ stimulus and $-245^{\circ}$ for the pp $1^{\circ}$ stimulus. Thus, $B S$ responses are close to being in phase with tilt at lower frequencies and in counter-phase at the highest frequency tested (see stickman representations of the responses in Fig. 3Bb and c). The coherence curves of the four peak-peak tilt amplitudes resembled each other. Coherence values were found to be around 0.7 in the low frequency range $(0.017-0.182 \mathrm{~Hz})$ and about 0.5 at higher frequencies up to approximately $1 \mathrm{~Hz}$, whereas above $1 \mathrm{~Hz}$ they fell below 0.5 . The averaged $L S$ responses to tilt stimulus (Tilt-to-LS FRFs and coherence curves; Fig. $3 \mathrm{Ab}$ ) showed similar characteristics as compared to the $B S$ responses.

The averaged TS responses to tilt stimuli (Tilt-to-TS FRFs and coherence curves) are shown in Fig. 3Ac. The gain and phase curves are again similar in shape to those of $B S$ in Fig. 3Aa, with the gain curves also showing the amplitude non-linearity. However, there are two differences, indicating that the TS responses are not determined by the ankle joint responses alone. First, in the low frequency range up to $0.3 \mathrm{~Hz}$, TS gain is lower than $B S$ gain, indicating that the trunk was maintained closer to vertical than the body COM (compare stickman in Fig. $3 \mathrm{Bb}$ ). Second, at frequencies above $0.5 \mathrm{~Hz}$, the TS gain values are higher than those of BS. Furthermore, the TS phase lag increases to a greater extent with increasing frequency compared to the $B S$ phase. In the stickman representation for $2.2 \mathrm{~Hz}$ (Fig. 3Bc), TS excursion is relatively larger than $B S$ and is in counter-phase to $B S$. Coherence is approximately 0.5 up to a frequency of $0.8 \mathrm{~Hz}$, with the exception of a dip at about $0.3 \mathrm{~Hz}$. Above $0.8 \mathrm{~Hz}$, coherence values for the pp $4^{\circ}$ stimulus decrease below 0.5 and even more so for $\mathrm{pp} 2^{\circ}$ and $1^{\circ}$ stimulus.

The ratio of TS gain to $L S$ gain and the difference between TS phase and $L S$ phase for each stimulus amplitude and stimulus frequency is shown in Fig. 3Ba. The four gain ratio curves are essentially constant across frequency for stimulus frequencies below $0.5 \mathrm{~Hz}$. With increasing stimulus amplitude, the gain ratios slightly decrease from 0.8 for $\mathrm{pp} 1^{\circ}$ to 0.5 for $\mathrm{pp} 8^{\circ}$. At frequencies above $0.5 \mathrm{~Hz}$, the ratio curves increase and clearly exceed unity. Here the trunk sway is larger as compared to the leg sway. At the lowest tilt frequency, trunk and leg responses to support surface tilt are essentially in phase. With 
increasing tilt frequency the trunk shows an increasing phase lag with respect to the leg sway response, which reaches $-100^{\circ}$ at a tilt frequency of $2.2 \mathrm{~Hz}$.

The results of the control experiment, where the tilt stimuli were applied to the leg segment with the rotation axis through the hip joints, are given in Fig. 4A. Shown are the averaged FRFs of two subjects from the leg segment stimuli to the trunk responses ( $L S_{S T I M}-t o-T S$ FRFs) for three stimulus amplitudes ( $\mathrm{pp} 1^{\circ}, \mathrm{pp} 2^{\circ}$, and $\mathrm{pp} 4^{\circ}$ ). The gain curves are similar in shape as the gain curves of Tilt-to-BS, Tilt-to-LS and Tilt-to-TS FRFs in Fig. 3Aa-c, showing an amplitude non-linearity. The phase curves show a small phase lead in the low frequency range and an increasing phase lag in the mid frequency range, which has also been observed in the Tilt-to-BS, Tilt-to-LS FRFs and Tilt-to-TS FRFs. The gain and phase curves at frequencies above $1 \mathrm{~Hz}$ are probably not reliable, since the fixation of the subjects on the platform was not rigid enough to analyze the small amplitude responses at high frequencies.

\section{Modeling and model simulations}

Based on a previous study (Hettich et al., 2011), a refined multi-sensory feedback control model for hip and ankle joint is proposed in this study to interpret the complex pattern of the $B S, L S$ and $T S$ gain and phase curves for four different tilt stimulus amplitudes. The results of the model simulations will be compared to human sway responses and the robustness to parameter variations of the control model will be tested in a sensitivity analysis.

\subsection{Hip and ankle joint control model}

In the current study, the DEC concept was extended from SIP to DIP biomechanics by adding a hip joint to the ankle joint and a separate DEC control module for balancing the trunk on the hips (extended DEC model; Fig. 5). The equations that define the DIP mechanics are given in the Appendix A. The lower part of Fig. 5 shows the ankle joint control (Ankle Module) and the upper part shows the hip joint control (Hip Module). The principles of the extended DEC model are explained first for the Ankle Module and then for the Hip Module. One may distinguish the following three components:
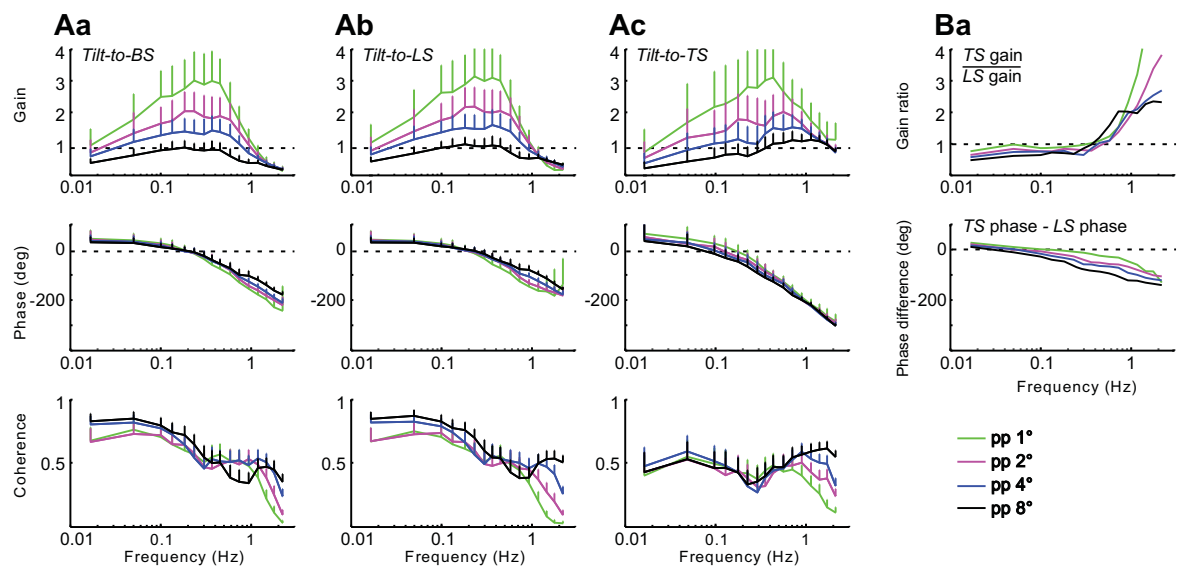

Bb

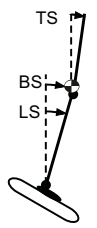

Bc

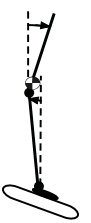

Fig. 3. Averaged tilt responses of six human subjects. Shown are gain, phase and coherence curves over frequency for the four peak-peak stimulus amplitudes and their upper 95\% confidence limits. Gain of zero would indicate ideal tilt compensation, a gain of unity that the evoked body or segment excursion equals the tilt excursion. The phase gives the temporal relation of stimulus and response. Coherence is a measure of the frequency dependent signal-to-noise ratio. (Aa) BS responses to Tilt (Tilt-to-BS FRFs). (Ab) LS responses to Tilt (Tilt-to-LS FRFs). (Ac) TS responses to Tilt (Tilt-to-TS FRFs). (Ba) Ratio curves of TS gain to $L S$ gain and difference curves between $T S$ phase and $L S$ phase. Stickman figures show largest excursion angles and their phase relation for the lowest tilt frequency, $0.017 \mathrm{~Hz}$ (Bb; angles are drawn 15 times larger as measured responses) and for the highest tilt frequency, $2.2 \mathrm{~Hz}$ (Bc; 4000 times larger). 
(1) Box Body. The box represents the plant and contains the biomechanics of the upright body and the two external disturbances having impact on it during support surface tilt: the gravitational ankle torque $T_{A_{-} \text {grav }}$ resulting from $B S$ excursion and the foot-space tilt angle FS defined by the tilt stimulus (assuming firm contact of the feet on the support surface). Note that FS tends to take the body somewhat with the tilt due to intrinsic musculoskeletal stiffness and viscosity (omitted in Fig. 5 for simplicity). The input to the box Body is the Foot-Space Tilt angle FS and the ankle torque $T_{A}$ from the ankle controller (box $C_{A}$ ). The outputs are the physical variables Leg-Space angle $L S$, Leg-Foot angle $L F$ and COP shift under the feet. The physical variables are measured by corresponding sensory organs (see Introduction). The ankle proprioceptive sensors measure the leg-foot angle If and its first derivative if (box $P R O P_{A}$ ) and torque sensors measure ankle torque $T_{A}$ (box TORQUE). The frequency characteristics of the proprioceptive signals and of the vestibular signals in the vertical planes (derived from the Hip Module) were taken to be ideal (Mergner, Schweigart, \& Fennell, 2009).

(2) Local Negative Feedback loop. The local feedback loop transforms the difference between desired and actual joint position into the torque command $T_{A}$ using a PD controller (P, proportional, and $\mathrm{D}$, derivative factor; box $C_{A}$ ). The muscle actuation dynamics are not modeled explicitly and are included in the controller dynamics. The controlled variable is the body-space angle $B S$, represented internally by the signal $b s$. It is obtained using (a) the foot-space angle signal $f s$ to transform the leg-foot angle signal lf into the leg-space angle signal $l s^{\prime \prime}$, and (b) the trunk-space angle signal ts to calculate bs (box COM; details in Appendix A). With appropriately adjusted parameters, the negative feedback of the bs signal and the controller represent a servo-mechanism that makes the actual movement correspond to the desired movement such that no feed forward of the plant dynamics is required (e.g., through an inverse of plant dynamics; Mergner, 2004; Mergner, 2010). However, the servo-mechanism performs desired movements accurately only in the absence of external disturbances such as gravity.
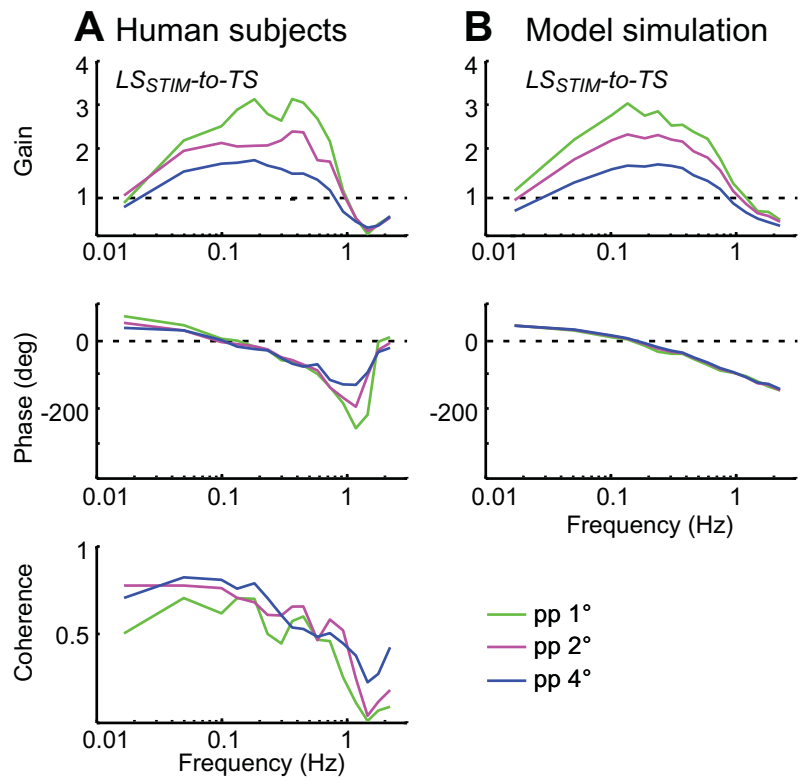

Fig. 4. Experimental and corresponding simulation results of control experiment. In the experiments, subjects' legs were fixed to a mechanical setup on the motion platform. PRTS tilt stimulus was applied to $L S$ with the rotation axis passing through subjects' hip joints. Shown are gain, phase and coherence curves of $L S_{S T I M}$-to-TS FRFs for pp $1^{\circ}, 2^{\circ}$ and $4^{\circ}$ tilt amplitudes. (A) Mean curves of two subjects. (B) Simulation results. 
(3) Disturbance estimation and compensation (DEC) feedback loops. The manifold of disturbance scenarios during upright stance can be categorized into four relevant external disturbances: (a) support surface translational acceleration, (b) field forces (e.g., gravity), (c) contact forces (e.g., push) and (d) support surface tilt. External impact on the body is decomposed into estimates of these four external disturbances based on sensory information (estimates in boxes Translational Acceleration, Gravitational Ankle Torque, External Ankle Torque and Foot-Space Tilt; Mergner, 2010). The estimates are used in feedback loops to directly compensate the corresponding disturbances. During the here considered support surface tilts, the disturbances (b), (c) and (d) are relevant. The External Ankle Torque estimate (c) may help to compensate for the inter-segmental coupling torque exerted by trunk accelerations on the leg segment (see Appendix A). Vestibular derived signals of trunk-space angular velocity $\dot{t s}$ and of angular position with respect to the gravitational vertical ts are used together with corresponding proprioceptive signals for the Foot-Space Tilt estimate and the Gravitational Ankle Torque estimate, respectively (see Appendix A). In addition, ankle torque information, thought to be derived from Golgi-Tendon organs and COP sensing pressure receptors in the feet, is used in the External Ankle

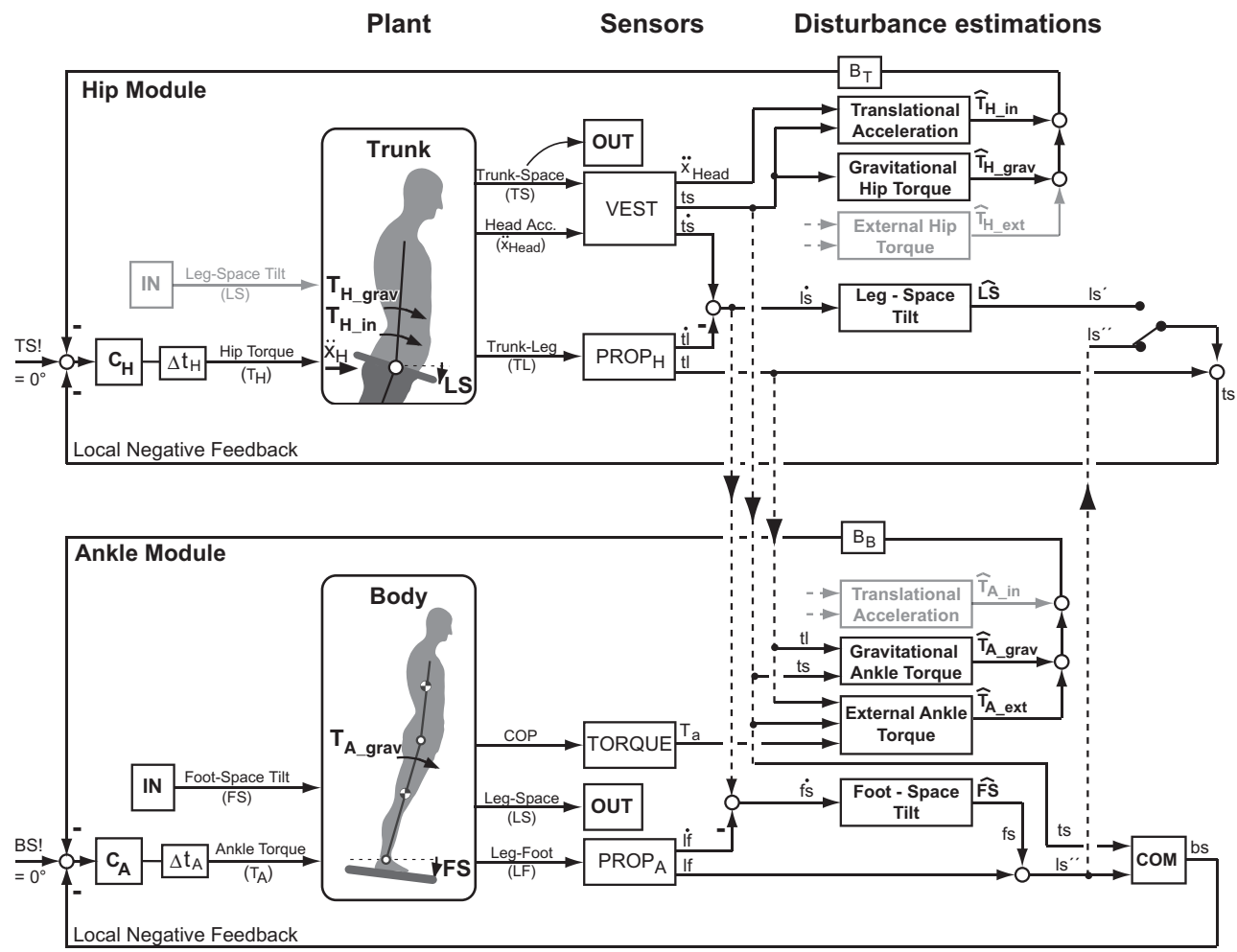

Fig. 5. Extended DEC model. It combines whole-body balancing on ankle joints (control by Ankle Module) and trunk balancing on hip joints (control by Hip Module). The input to the model simulations is Foot-Space Tilt (FS) angle and the measured outputs are Leg-Space (LS) and Trunk-Space (TS) angles (boxes IN and OUT). Also shown are the boxes of the activated disturbance estimates (inactive estimates are gray). The set point signals for the two modules are desired body-space angle BS! and desired trunk-space angle TS!. Sensory information used by the estimates is derived from the vestibular system (box VEST), hip joint proprioception $\left(P R O P_{H}\right)$, ankle joint proprioception $\left(P R O P_{A}\right)$, and ankle torque sensor (TORQUE). Boxes ' $C_{H}$ ' and ' $C_{A}$ ' indicate the neural controllers for the hip and ankle modules and ' $\Delta t_{H}$ ' and ' $\Delta t_{A}$ ' the corresponding lumped time delays. Boxes ' $B_{T}$ ' and ' $B_{B}$ ' provide transformations from torques into angle equivalents. Note that uppercase letters for angles and angular velocities represent physical variables and lowercase letters the corresponding internal representations. Disturbance estimates are marked by '^’. In the control experiment simulations, the stimulus input is Leg-Space Tilt (LS) angle (gray box IN) with switch setting on $I s^{\prime}$. 
Torque estimate. All estimations include signal processing in the form of a central detection threshold and gain scaling (see Appendix A). This processing together with summation effects across the network of sensory pathways are able to explain the sensory re-weightings that are observed in human experiments upon changes in stimulus modality and amplitude (see Fig. 7 in Maurer et al., 2006).

The Hip Module (upper part of Fig. 5) is analogous in structure to the Ankle Module. The box Trunk contains the biomechanics of the trunk balancing on the legs, with the hip torque $T_{H}$ as input (the input Leg-Space Tilt angle $L S$ was only used in the control experiment). The outputs are the physical variables Trunk-Space angle TS, head translational acceleration $\ddot{x}_{\text {Head }}$ and Trunk-Leg angle TL. The hip proprioceptive sensors measure trunk-leg angle $t l$ and its first derivative $\dot{t} l$ (box $P R O P_{H}$ ) and the vestibular sensors measure $t s, \dot{t s}$, and head translational acceleration $\ddot{x}_{\text {Head }}$ (box VEST).

During support surface tilt, the disturbance estimates (a), (b), and (d) are relevant for the hip control. That is, also the Translational Acceleration estimation contributes, which accounts for the fact that during leg rotations, the hip joints follow an eccentric rotation, which is associated with a tangential acceleration. Furthermore, the leg-space information used in the Local Negative Feedback loop can be thought to be derived from two available leg-space signals in the model. One is obtained within the Hip Module from the Leg-Space Tilt estimate in the form of the $l s^{\prime}$ signal. The other is derived in the Ankle Module by combining the signal from the Foot-Space Tilt estimate with the ankle proprioception in the form of the $l s^{\prime \prime}$ signal.

\subsection{Parameter identification}

Modeling and simulations were performed using Matlab/Simulink (The Math Works Inc., Natick, USA). Control parameters were chosen such that one and the same parameter set (Table 1) sufficed to reproduce subjects' sway responses across the different tilt stimulus frequencies and amplitudes. In the framework of the DEC concept, parameter identification is constrained in several respects (Mergner, 2010). The DEC concept derives the controller parameters from the anthropometric measures of the subjects, assuming for the controller a proportional factor of $1 \mathrm{mgh}$ ( $\mathrm{m}$, body mass; $\mathrm{g}$, gravitational acceleration; $h$, COM height above the joint) and for the derivative part a value of $0.3 \mathrm{mgh}$ in the Ankle Module and $0.2 \mathrm{mgh}$ in the Hip Module. With (i) the previously identified lumped time delay, (ii) the value for the human body inertia, and (iii) unity gain for the combined effect of reflexive and intrinsic feedback in the 'servo loop', the servo mechanism ensures that the actual movement corresponds to the desired movement (see Maurer et al., 2006; Mergner, 2010). In the presence of external disturbances such as gravity, the parameters of the controller and the servo-loop are assumed to remain constant when the DEC loops are added (Mergner, 2010). With these constraints, the parameter identification focused on the thresholds and the gain factors in the disturbance estimates. In order to reduce the number of parameters, which are optimized at a time, the optimization procedure was performed separately for the Ankle Module and the Hip Module.

The Matlab function 'fminsearch' with the Nelder-Mead simplex direct search algorithm from the 'Optimization toolbox' was used. The optimization procedure was set to minimize the sum of the squared error between model simulation and corresponding experimental data. Since the phase of the sway responses is mainly determined by the time delay in the model, which was assumed to be fixed, only sway response gain was used to calculate the simulation error. The error value was calculated across all four amplitude conditions and across all stimulus frequencies, where smoothed gain curves were used in order to deemphasize the influence of higher frequencies on the simulation error (compare Peterka, 2002). A global optimization approach was used, running the optimization from different starting values. Two different initial values were chosen for each parameter and the optimization was started at each combination of initial values. The result with the smallest overall error was chosen as the final result. The function 'fminsearch' was modified to allow for upper and lower limits of the parameters in order to reduce the computational cost. The bounds were set to allow only for plausible values. For example, the gain factors of the estimates had an upper limit of 0.95 , as these gain factors represent a degree to which the nervous system 'trusts' the estimate. It is unlikely that the gains in the estimates reach unity as the estimates are based on noisy sensory signals. For the Ankle 
Module, the optimization algorithm minimized the difference between the simulation and the corresponding experimental gain values of the Tilt-to-BS FRFs. The threshold values and gain factors of the Foot-Space Tilt and Gravitational Ankle Torque estimates were evaluated in the optimization procedure.

For the Hip Module, the optimization algorithm minimized the difference between experimental and simulated $T S^{\prime}$ to $L S^{\prime}$ gain ratios by adjusting the thresholds and gain factors of the Leg-Space Tilt and the Gravitational Hip Torque estimates. Here, the gain ratios were used instead of the Tilt-to-TS FRFs values mainly because simulation results appeared much more stable in the global optimization. One reason might be that the gain ratio is a relative measure of trunk to leg sway and is therefore less affected by errors in the estimation of the Ankle Module parameters. The considered frequency range in the Hip Module parameter optimization procedure was furthermore restricted to the frequency range of $0.016-0.7 \mathrm{~Hz}$, as parameter estimates became less stable when including higher frequency ranges. The restriction to the low and mid-frequency range is supported by the finding that sensory feedback control, which is the main focus of the current study, affects the trunk responses mainly in the low and mid-frequency range (Kiemel et al., 2008).

\subsection{Simulation results}

Model simulation results for the four PRTS stimulus amplitudes and both possible sources of the leg-space signal $l s\left(l s^{\prime}\right.$ and $l s^{\prime \prime}$ ) are shown in Fig. 6. The panels Aa-c and B give the results for the $l s^{\prime}$ signal from the Leg-Space Tilt estimate of the Hip Module (switch setting: $l s^{\prime}$ in Fig. 5). The shape of the simulation gain curves of the Tilt-to-BS', Tilt-to- $L S^{\prime}$ and Tilt-to-TS' FRFs (Fig. 6Aa-c) resembles the main characteristics of the human experimental results. Specifically, the simulation gain curves show the characteristic shape and the amplitude non-linearity, and the simulated phase curves show the increasing phase lag with increasing stimulus frequency (compare Fig. $3 \mathrm{Aa}-\mathrm{c}$ ). The simulations

\section{Aa}

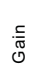
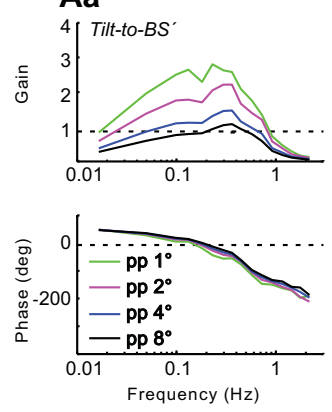

$\mathrm{Ca}$
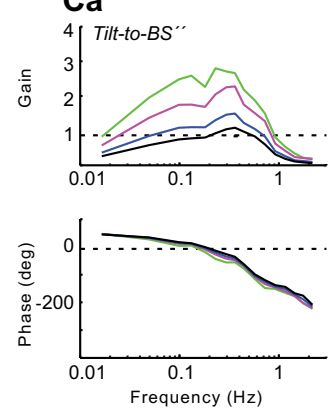

Ab
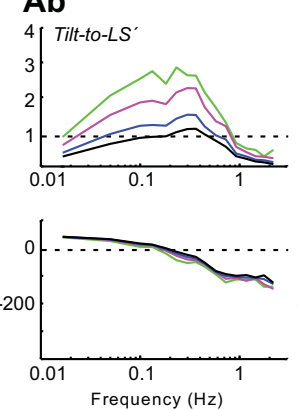

$\mathrm{Cb}$
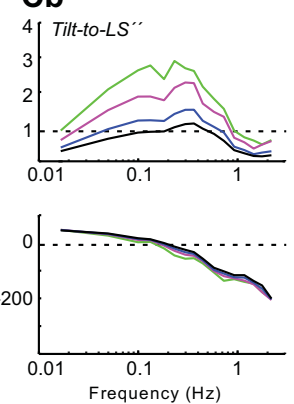

Ac
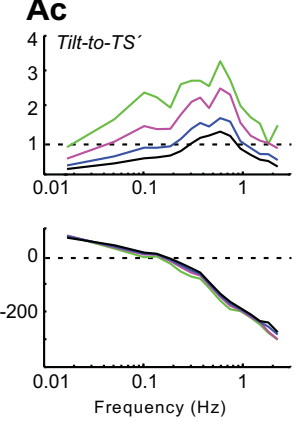

Cc
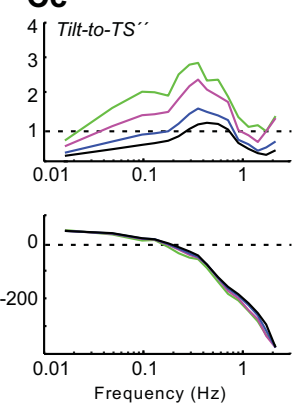
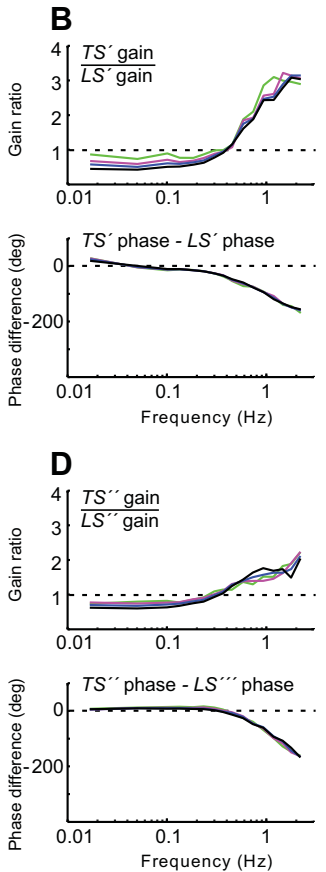

Fig. 6. Model simulations for the switch settings $l s^{\prime}$ and $l s^{\prime \prime}$ (compare Fig. 5). (Aa-c) Tilt-to-BS', Tilt-to-LS', and Tilt-to-TS' FRFs and (B) $T S^{\prime}$ to $L S^{\prime}$ gain ratio and phase difference curves obtained when using the leg-space signal $l s^{\prime}$ derived from the Leg-Space Tilt estimate in the Hip Module. (Ca-c) shows the corresponding FRFs and (D) the corresponding gain ratio and phase difference curves when using the $l s^{\prime \prime}$ signal 'up-channeled' from the Ankle Module. Comparable human experimental data are shown in Fig. 3. 
also reproduce the main characteristics of the trunk sway responses found in the human subjects expressed as the $T S$ to $L S$ gain ratios and phase differences (compare Fig. 6B with Fig. 3Ba). In the low frequency range up to $0.5 \mathrm{~Hz}$, the trunk segment sways less than the leg segment, as indicated by the gain ratios being below unity in both, simulation and experimental results. The simulations also reproduced the dependence of the gain ratio on tilt stimulus amplitude, where the gain ratio is largest for the smallest tilt stimulus amplitude. For frequencies above $0.5 \mathrm{~Hz}$, the $T S$ to $L S$ gain ratio curves of the simulation increase up to a value of 3 , and the phase differences increase and reach almost counter phase at about $2.2 \mathrm{~Hz}$, a finding that also resembles the experimental results.

In the simulation results shown in the panels $C$ and D of Fig. 6, the leg-space signal from the Foot-Space Tilt estimate of the Ankle Module was used for the simulation (switch setting: $s^{\prime \prime}$ in Fig. 5). The Tilt-to- $B S^{\prime}$ FRFs and the Tilt-to- $L S^{\prime}$ FRFs remained essentially the same as in the $l s^{\prime}$ setting (compare Fig. 6Ca-b with Aa-b). Discrepancies to the $l s^{\prime}$ simulations were only found in the high frequency range of the Tilt-to-TS' FRFs (Fig. 6Ac vs. Cc) and of the gain ratios (Fig. 6B vs. D). Here, gain values were smaller compared to the $l s^{\prime}$ simulation results. In the low and mid frequency range the trunk responses where in phase with the leg responses.

To address the question whether the similarity between the Tilt-to-TS and the Tilt-to-LS FRFs can also be explained by high intrinsic stiffness and viscosity in the hip joints, further model simulations were performed. High intrinsic hip stiffness can be caused by co-contraction of antagonistic muscles (van Soest, Haenen, \& Rozendaal, 2003). When increasing step-wise intrinsic stiffness and viscosity in the hip joint and decreasing accordingly the active (reflexive) stiffness and viscosity, simulation results showed that the sway amplitude of the trunk segment was larger than the sway amplitude of the leg segment. More specifically, the gain ratio curves were larger than unity in the low and mid frequency range (not shown), which is different from the experimental findings in Fig. 3Ba. Therefore, the intrinsic stabilization of the hip without sensory feedback control was structurally different compared to experimental observations.

Further model simulations were performed to test whether the control model would reproduce the findings of the control experiment (see Sections 2 and 3 and Fig. 4A). In these simulations the trunk segments' biomechanics was modeled as a SIP, with the rotation at the hip joints. The tilt stimulus sequence was applied to the leg segment, which represents the support base for the trunk (Leg-Space Tilt, $L S$, input in Fig. 5). Tangential acceleration of the hip joint $\left(\dot{x}_{H}\right)$ is absent here. In this experimental setup, the Hip Module represents the supporting joint and the switch was set such that the $l s^{\prime}$ signal was used for the control. The control parameters were obtained using the same optimization procedure as for the Tilt-to-BS FRFs, whereas the considered frequencies were restricted to $1 \mathrm{~Hz}$. The parameters are given in the Hip Module section of Table 1 in parenthesis. The simulation results resemble the experimental results as shown in Fig. 4. The obtained threshold values and gain factors were similar to the parameters found for the Ankle Module and different from the parameters found for the Hip Module in the freestanding condition.

\subsection{Sensitivity analysis}

To ascertain that fine adjustments of parameters were not critical for the model's control stability, a sensitivity analysis was performed. It characterizes the sensitivity of the model-predicted tilt responses to variations in the control parameters. To this end, each parameter was independently increased and decreased by $10 \%$. The resulting gain curves of the Tilt-to-BS' FRFs and of the $T S^{\prime}$ to $L S^{\prime}$ gain ratio curves were superimposed on the curves from the initial parameters.

The most relevant results of the sensitivity analysis are shown in Fig. 7. The figure repeats the gain curves of Tilt-to-BS' FRFs (Aa and $\mathrm{Ba}$ ) and the $T S^{\prime}$ to $L S^{\prime}$ gain ratios $\left(\mathrm{Ab}\right.$ and $\mathrm{Bb}$ ) for the pp $2^{\circ}$ and $8^{\circ}$ model simulations from Fig. 6, Aa and B, respectively. In Fig. 7Aa and b the controller parameters $\mathrm{P}$ and $\mathrm{D}$ and the time delay of the Ankle Module were increased and decreased by $10 \%$ of the identified values. These parameter variations affected mainly the Tilt-to-BS' FRFs and hardly the $T S^{\prime}$ to $L S^{\prime}$ gain ratio curves. However, the effects were relatively small compared to idiosyncratic differences between subjects, and control stability was not endangered. Similar weak effects were obtained when varying gain and threshold values of the disturbance estimates (not shown). Fig. 7Ba,b shows the results obtained when varying the corresponding control parameters of the Hip Module. There were no visible 


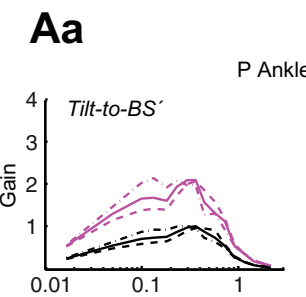

Ab

$29 \mathrm{~N} \mathrm{~m} \mathrm{rad}^{-1}$ )

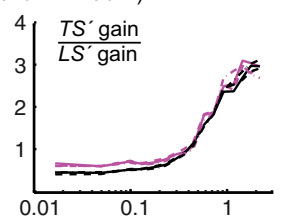

D Ankle $\left(189 \mathrm{~N} \mathrm{~m} \mathrm{~s}^{-1} \mathrm{rad}^{-1}\right)$
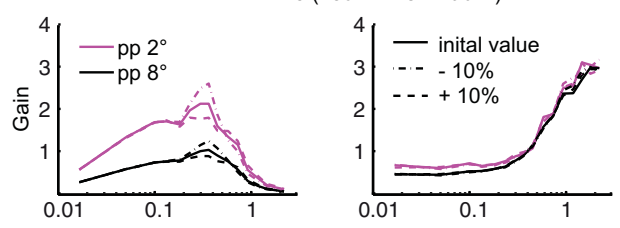

Time delay Ankle $(0.18 \mathrm{~s})$
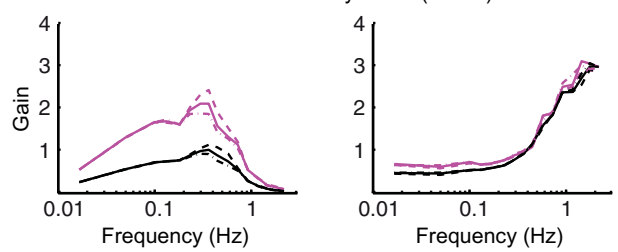

$\mathrm{Ba}$

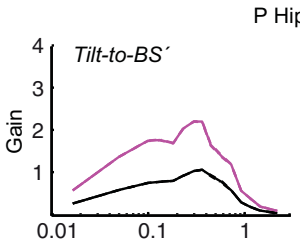

Bb

Hip (144 $\left.\mathrm{N} \mathrm{m} \mathrm{rad}^{-1}\right)$

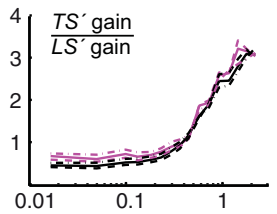

D Hip $\left(29 \mathrm{~N} \mathrm{~m} \mathrm{~s}^{-1} \mathrm{rad}^{-1}\right)$
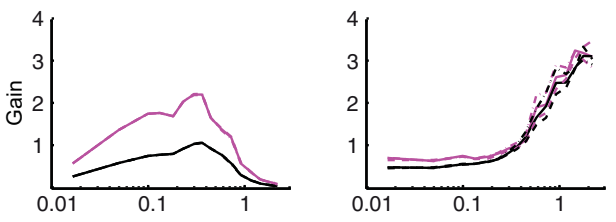

Time delay Hip $(0.07 \mathrm{~s})$
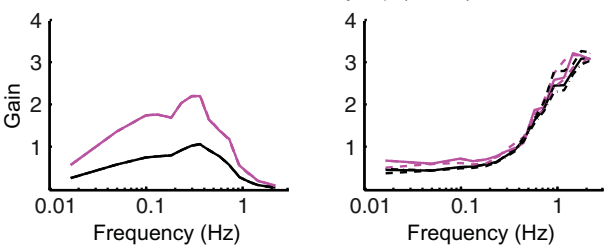

Fig. 7. Sensitivity analysis using model parameter variations (switch setting $l s^{\prime}$ ). Shown are simulated gain and gain ratio curves for the pp $2^{\circ}$ (magenta) and pp $8^{\circ}$ (black) PRTS tilt stimuli obtained with the identified parameter values (solid curves) and the effects obtained with $10 \%$ increased and decreased parameter values (dashed and dashed-dot curves, respectively). (A) Effects of Ankle Module control parameter variations on Tilt-to-BS' FRFs (Aa) and $T S^{\prime}$ to $L S^{\prime}$ gain ratios (Ab). (B) Effects of the Hip Module control parameter variations on Tilt-to- $B S^{\prime}$ FRFs $(\mathrm{Ba})$ and $T S^{\prime}$ to $L S^{\prime}$ gain ratio curves $(\mathrm{Bb})$.

effects on the Tilt-to-BS' FRFs and only small changes in the $T S^{\prime}$ to $L S^{\prime}$ gain ratio curves for all test parameters.

\section{Robot simulations}

The robot simulations adopted an approach that was successfully used in previous studies on ankle joint balancing with SIP biomechanics (custom made humanoid biped PostuRob I; Mergner, Huethe, Maurer, \& Ament, 2006; Mergner et al., 2009). A new robot was built (Posturob II; Fig. 8A), which contains two actuated hip joints and ankle joints.

\subsection{PostuRob II}

PostuRob II was constructed with human-like anthropometric parameters. It consists of trunk, leg and feet segments of aluminum, interconnected by hinge joints resembling the hip joints and the ankle joints. The legs' segment length, mass (both legs) and COM height above the ankle joint amounted to $0.86 \mathrm{~m}, 20 \mathrm{~kg}$, and $0.45 \mathrm{~m}$, respectively. The trunk's segment weight and trunk COM height above hip joints amounted to $30 \mathrm{~kg}$ and $0.28 \mathrm{~m}$, respectively. Total height above the ankle joints was $1.67 \mathrm{~m}$ and the weight without feet was $50 \mathrm{~kg}$. Signals from mechatronic sensors (vestibular, joint torque, joint angular position and velocity) were input into a real time PC, where the control model was executed as a compiled Simulink model (Real-Time Windows Target, The MathWorks Inc., Natick, USA). The vestibular sensor processed accelerometer and gyrometer signals and delivered the signals trunk angular velocity, angle with respect to the gravitational vertical, and linear acceleration in the 
sagittal plane (Mergner et al., 2009). The torque commands for hip and ankle joints actuated artificial pneumatic 'muscles' (FESTO AG \& Co.KG, Esslingen, Germany; Typ MAS20). An inner torque control loop ensured that the actual torque matched the desired torque.

\subsection{Robot experimental results}

The extended DEC model (Fig. 5) was re-embodied into Posturob II and the controller parameters were adjusted to the anthropometric parameters of the robot. During the experiments, the robot was standing freely on the motion platform. Robot experiments were performed in essentially the same way as the human experiments and also the analyses of the responses were the same. A video showing PostuRob II balancing a pp $8^{\circ}$ PRTS tilt stimulus sequence can be found at www.posturob.uniklinikfreiburg.de. Additionally, the parameters of the disturbance estimates were slightly adjusted to match the robot experimental data to the human experimental data. These small adjustments can be assigned to the imperfections of the real world environment with mechanical dead zones, inaccuracies of the sensory signals, etc. With these small adjustments, the main characteristics of the gain and phase curves of the Tilt-to-BS FRFs (Fig. 8Ba) were similar to those obtained from the corresponding model simulations (Fig. 6Aa) and human data (Fig. 3Aa) apart from a gain dip around $0.2 \mathrm{~Hz}$ with the smallest tilt amplitude ( $\mathrm{pp} 1^{\circ}$ ), which also was seen in similar form in single responses of subjects. Also the robot's Tilt-to-LS and Tilt-to-TS FRFs resembled the human and model simulation data (not shown) as well as the $T S$ to $L S$ gain ratio and phase difference curves (Fig. $8 \mathrm{Bb}$ ).

The exchange of the sensory signals between the Ankle and Hip Modules in the model (Fig. 5) produces coordination between hip and ankle joints as an automatic postural reaction that keeps the body COM vertical above the ankle joints. Generally, this applies when one or several of the four external disturbances have impact on the body and also when the disturbance is self-produced. To show an example for the resulting coordination, a 'voluntary' forward trunk bending of the robot is shown in

A

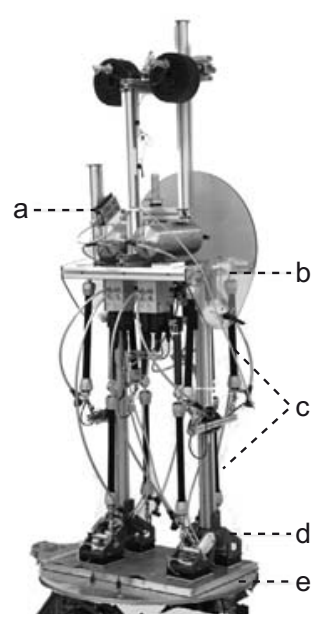

\section{$\mathrm{Ba}$}

듕
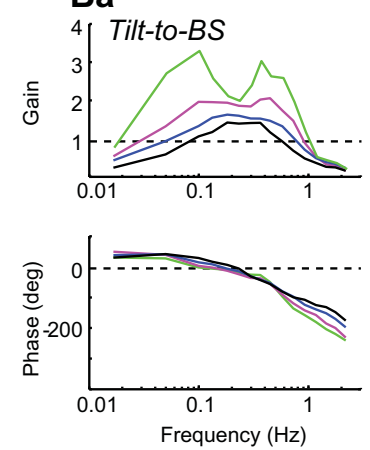

C

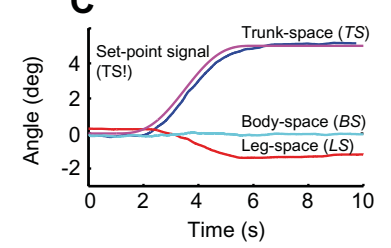

\section{$\mathrm{Bb}$}
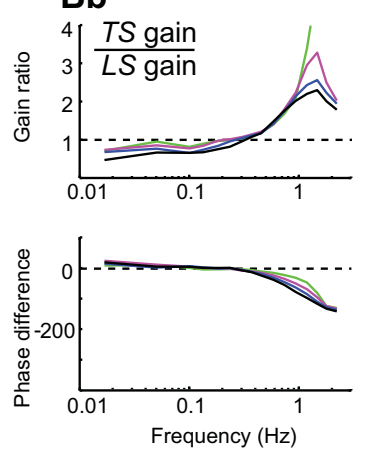

$-\mathrm{pp} 1^{\circ}$

$-\mathrm{pp} 2^{\circ}$

Fig. 8. PostuRob II and robot experimental results obtained with the extended DEC model. (A) PostuRob II standing on a motion platform (e). The robot consists of trunk, leg and foot segments, interconnected by the hip joints (b) and ankle joints (d). An artificial vestibular system (a) and joint angle sensors provide sensory information. Artificial pneumatic muscles (c) generate the joint torques. (B) Gain and phase curves for Tilt-to-BS FRFs (Ba) and TS to $L S$ gain ratio and phase difference curves (Bb). (C) Time series of a voluntary trunk bending (set point signal, TS!). The TS forward lean is associated with an automatic $L S$ backward lean, stabilizing the body COM in space $(B S)$. 
Fig. 8C. It is produced using a position trajectory as set point signal for the Hip Module (TS! signal) and an interaction between sensed and predicted gravitational torque compensation was used by which the trunk bending becomes more accurate (see Mergner, 2010). The trunk bending is associated with a backward lean of the leg segment, which emerges from the exchange of sensory signals between Hip Module and Ankle Module.

\section{Discussion}

This study investigated human balancing responses in the hip and ankle joints to support surface tilts and proposed a model of the underlying sensory feedback control. The model was based on the DEC concept, in which estimates of external disturbances are derived from sensory signals and used to compensate for the disturbances instead of using the 'raw' sensory signals directly. In the current study, the DEC concept was extended to cope with DIP biomechanics with human anthropometrics, human-like time delays and mechanical interactions between the body segments. The extended DEC model applies a modular structure in which each joint is controlled with a separate DEC Module. The simulation results demonstrated that the hip-ankle coordination results observed in humans can be reproduced when including interconnections between the DEC Modules on the sensory level. Implementing and testing the proposed model in a humanoid robot demonstrated its functionality and stability in a real world environment as a proof of principle. Model and robot simulations are in good agreement with the human experimental results, suggesting that the model describes important aspects of the human balancing.

The model contains some differences compared to a model presented in a preliminary note (Hettich et al., 2011), where only one stimulus amplitude was used and several problems remained unsolved. One difference is that the present model does not involve an integrative factor (I) in the PD controller for ankle and hip joints, i.e., a PD controller was used instead of a PDI controller (explanation in Appendix A). Another difference is the alternate use of either the $l s^{\prime}$ or the $l s^{\prime \prime}$ signal. Further, minor differences include a larger time delay value for the ankle joint control and changes in the gain factors and thresholds in the disturbance estimates. As shown in the sensitivity analysis (4.4), however, the effects of such variations affected the results only marginally.

Human sway responses to support surface tilts, often expressed as gain curves, are known to show an amplitude non-linearity (Maurer et al., 2006; Peterka, 2002). It was suggested that this nonlinearity is related to detection thresholds in the disturbance estimates (Maurer et al., 2006), comparable to those observed in psychophysical experiments on vestibular and neck-proprioceptive self-motion perception (Mergner et al., 1991). Such thresholds have also been discussed in relation to sway responses to visual scene motions (Peterka \& Benolken, 1995). Due to the thresholds, the sway responses evoked by small stimuli are less compensated and therefore relatively larger as compared to sway responses evoked by large stimuli.

To understand the specific implementation of the velocity threshold in the Foot-Space Tilt estimate, several aspects need to be considered. First, the thresholds in the estimates are thought to have a noise reducing function, when the estimated disturbance is absent or small (Mergner et al., 1991). Second, the vestibular system is a major contributor to the overall noise in the posture control mechanism, with an approximately 10 times higher noise level as compared to the proprioceptive system (Mergner et al., 2009; van der Kooij \& Peterka, 2011). Third, in accordance with the DEC concept, vestibular signals are combined with proprioceptive signals to obtain an estimate of the support surface angular velocity, where the large noise contribution of the vestibular system adds to the small noise contribution of the proprioceptive signal ('down-channeling' of vestibular and proprioceptive signals; Mergner et al., 1997). When implementing the threshold in the tilt estimate, the contribution of vestibular noise in the feedback mechanism becomes dependent on the actual movement of the support surface, rather then on the sway of the body signaled by the vestibular system. This mechanism therefore automatically reduces the noise contribution from the vestibular system whenever the signal of the tilt estimate is small or zero, i.e., on a firm and stationary support surface. The noisy space reference of the vestibular system only contributes, when it is actually needed to account for the moving support surface (Fig. 3C in Mergner et al., 2009). The combination of this tilt estimate with the 
proprioceptive leg-foot signal If then results in a context dependent, automatic sensory re-weighting mechanism for the $l s^{\prime \prime}$ signal. When the support surface is stationary the if velocity signal and the down-channeled is velocity signal cancel each other and the combined noise remains below the threshold of the estimate. In this case, the $l s^{\prime \prime}$ signal is fully determined by the proprioceptive signal If. With increasing support tilt velocity, the proprioceptive signals tend to cancel each other and the contribution of the vestibular signal to $l s^{\prime \prime}$ increases.

In the current study, we addressed the question whether the improved leg-space signal $\left(l s^{\prime \prime}\right)$ is also used to control the upper body. The rationale is to reduce the noise level in the Hip Module whenever the support surface is stationary. Transmitting signals from lower joints to higher joints, often referred to as 'up-channeling' has been found in single neuron recordings in a cervical ascending spinal tract of cats (Coulter, Mergner, \& Pompeiano, 1976). In humans, the up-channeling mechanism has also been suggested before on the basis of experiments on human self-motion perception (Mergner \& Rosemeier, 1998) and postural responses (Creath, Kiemel, Horak, \& Jeka, 2008). In model simulations either the $l s^{\prime}$ signal or the up-channeled $l s^{\prime \prime}$ signal could potentially be used (see switch in Fig. 5). The simulation results resemble the human results for both switch settings (Fig. 6) such that we cannot distinguish between the two possible sources of the ls signal in the Hip Module based on the current data.

The proposed DIP control model assumes a modular control architecture, where the hip and ankle joints are controlled by two separate, but interconnected DEC Modules. To test this assumption, model simulations of the control experiment were performed. In these simulations, the gain and phase curves produced by the Hip Module resembled the main characteristics of the gain and phase curves of the human experimental results of the control experiment (compare Fig. 4B with A). The identified gain factors and threshold values of the disturbance estimates (Table 1; Hip Module values in parenthesis) differ from the identified Hip Module parameters during the free-standing condition. The reason for this difference is currently unsolved. Interestingly, the parameters of the Hip Module in the control experiment were similar to those of the Ankle Module in the free-standing condition. One speculative explanation is that in the control experiment, the hip joint represents the supporting joint, comparable to the ankle joint in the free-standing condition. It seems that the thresholds and gain factors in the Hip Module depends whether the disturbance is external and unpredictable (such as a PRTS tilt stimuli) or self-produced (such as the leg segment rotation in free-standing). This dependence on the postural configuration will be a topic of future work.

In a recent study, Boonstra et al. (2013) argued that in a system with the hip-ankle DIP biomechanics two disturbances should be applied in order to identify the relationship between hip and ankle joint angles and joint torques. Our approach differs in that we are not identifying a multivariate linear system, but we are using FRFs to describe the system responses to specific stimuli. In particular, since the present study describes a non-linear system, the FRFs were calculated separately for each of the stimulus amplitudes. These FRFs were then used as a means to compare the human experimental results with the model simulations and robot experimental results.

Concerning related previous studies, Goodworth and Peterka (2010), Goodworth and Peterka (2012) investigated human reactive balancing using a feedback control model with hip and ankle joints and an additional joint at L4/L5. A direct comparison of their model with the extended DEC model in the present form is not possible, because (a) the double support by the two legs in the frontal plane involves different biomechanics (e.g., with parallel-leg stance, the pelvis cannot be rotated in the frontal plane, but only translated; trunk rotations occur mainly in the lower vertebral column), and (b) the control parameters of the model of Goodworth and Peterka (2010) are different for each tilt amplitude, whereas the extended DEC model is valid without parameter changes.

Suzuki, Nomura, Casadio, and Morasso (2012) investigated DIP stabilization during unperturbed stance using a feedback control model for the sagittal plane and suggested an intermittent feedback control. The authors argued that human-like time delays and gains in the feedback loops speak against a continuous feedback (Suzuki, Nomura, \& Morraso, 2011; Suzuki et al., 2012). In their mainly engineering-inspired control model, the coordination arises by selecting different combinations of active hip and ankle torque in terms of a finite-state machine. A comparison of their results with the present work is problematic, because this would imply comparing spontaneous sway data, which presumably reflect to a large extent unknown internal noise and its interaction with the control system (Maurer \& 
Peterka, 2005), with responses to external, well defined stimuli. Only the latter allow detailed inferences regarding the control mechanism (van der Kooij et al., 2005). The simulations presented in the current study and those of Goodworth and Peterka (2010), Goodworth and Peterka (2012), where the amplitude non-linearity is reflected in the FRFs, show that models with continuous feedback control of the hip-ankle DIP biomechanics with human-like time delays and feedback gains can give valid descriptions of human balancing.

The extended DEC model may not necessarily be the only valid description of the here obtained human sway responses. We hold, however, that the extended DEC model is currently unique with respect to several aspects:

(1) Correspondence between human and model data. The main argument is the good correspondence that was obtained in the present study between the human experimental data and the model as well as robot simulation data for both, the ankle responses and the hip responses. To the best of our knowledge, the extended DEC model is currently the only model, which describes a mechanism by which the amplitude non-linearity and the hip-ankle coordination emerges with one set of model parameters. The extended DEC model tolerated human-like time delays and mechanical interactions between body segments. The mechanical interaction applied to the hip joint torque that results from tangential accelerations of the hip, which can be thought to be covered by the Translational Acceleration estimate in the Hip Module. In the Ankle Module, the mechanical interaction that arises from trunk rotations is largely neutralized by whole-body COM control, this possibly with the help of the External Ankle Torque estimate (see Hettich et al., 2011).

(2) Biological feasibility. From neurophysiological and psychophysical studies it is known that the sensory information used in the model is available in the central nervous system (see Introduction). Furthermore, the sensitivity analysis and the robot experiments demonstrate a humanlike robustness in that the control is stable in spite of inaccuracies of the sensors and across considerable changes in the control parameters. Finally, the low loop gain results in an inherent soft mechanical compliance, which again is observed in humans.

(3) Simplicity of the control mechanism. The extended DEC model of Fig. 5 represents a simple and parsimonious control mechanism, where the computational effort is small as compared to engineering solutions that involve Kalman filters and run an efference copy signal through a full dynamic model of the body (Kuo, 2005; van der Kooij et al., 1999). The simplicity also applies to voluntary movements, where disturbance compensation involves fusion of the sensorderived disturbance estimates with learned predictions of these estimates derived from an internal model of body kinematics (Mergner, 2010).

(4) Emergence of the hip-ankle coordination. In the extended DEC model, the hip-ankle coordination emerges automatically as a posture control response to support surface tilt, when the default function is to maintain equilibrium without effort and voluntary interference (set point signals $B S$ ! and TS! are zero). Noticeably, this does not mean that subjects cannot modify actively the tilt compensations (e.g., decide to move with the platform) or superimpose active movements (via corresponding BS! and TS! set point trajectories; see Mergner, 2010). We asked whether the hip-ankle coordination produced by the extended DEC model would also occur as an automatic postural adjustment in other behavioral situations, for example during voluntary trunk bending, where the coordination has repeatedly been investigated in humans (e.g., Massion, 1992; Vernazza-Martin et al., 2006). Corresponding simulations using the extended DEC model were in agreement with the human experimental results reported in these studies (see robot response in Fig. 8C).

(5) Double task of trunk segment control. The principle of a whole-body COM balancing by the Ankle Module allows for independent motion in the hip joint without losing balance. The extended DEC model reproduced the human relatively more upright orientation of TS as compared to $L S$ and $B S$ in the low and mid-frequency range (see stickman in Fig. $3 \mathrm{Bb}$ ). This more upright position is in agreement with the human balancing behavior observed in a previous study using sinusoidal tilts (Mergner, Schweigart, Fennell, \& Maurer, 2009). The trunk balancing in the previous study further improved when subjects used in addition visual information and thereby stabilized the head position in space better. 
(6) Modularity of the control architecture. The extended DEC model used a separate DEC Module for balancing the trunk on the hip. This can be generalized to a modular control architecture where each DoF is controlled by its own DEC Module. The interconnection between modules is hierarchical by distinguishing in each joint control a buttressing link (often also the weight bearing link) and a moving link. In this concept, the moving link may actually consist of a stack of further links and the DEC Module controls the resulting COM. Thus, despite of changes in the geometry of the above links, each DEC Module can work as if it were controlling a SIP (Lippi, Mergner, \& Hettich, 2013).

The modular architecture appears to be human-like and to provide better control stability than monolithic control architectures (e.g., Brooks, Breazeal, Marjanovic, Scassellati, \& Williamson, 1999). With the monolithic architecture, small local errors may generalize and thus endanger the control stability of the whole system. In contrast, in a modular architecture, restrictions of one joint (e.g., joint immobilization in a human by a plaster) can be compensated by other joints. Furthermore, the modular architecture easily allows extending the control by further increasing the number of DoF. The complexity of the control then increases merely linearly with the number of DoF. This notion was ascertained in model simulations where the two-joint model was extended to a four-joint model by including knee and neck joints and will be the focus of future research.

\section{Acknowledgements}

G.H. is supported by the Konrad-Adenauer-Stiftung. The study was supported by the European Commission (FP7-ICT-600698 H2R).

\section{Appendix A}

\section{A.1. Model parameters}

Table 1 gives the anthropometric and the model parameters used for simulation. Anthropometric parameters were calculated based on the subjects' mean mass, height, relative mass distribution, and segment length tables (Winter, 1990).

\section{A.2. Model of human biomechanics}

The biomechanics of human upright stance was modeled as a double inverted pendulum (DIP) in the sagittal plane. The biomechanical model can be linearized using the small angle approximation, assuming that the subject is maintaining his upright position close to the vertical. Joint torque can then be expressed by the following equations for hip torque

$$
T_{H}=\left(J_{T}+m_{T} h_{T}^{2}+m_{T} l_{L} h_{T}\right) \ddot{L}+\left(J_{T}+m_{T} h_{T}^{2}\right) \ddot{T} L-\left(m_{T} g h_{T}\right) L S-\left(m_{T} g h_{T}\right) T L
$$

and ankle torque $T_{A}$

$$
\begin{aligned}
T_{A}= & \left(J_{L}+J_{T}+m_{L} h_{L}^{2}+m_{T}\left(l_{L}^{2}+h_{T}^{2}+2 l_{L} h_{T}\right)\right) \ddot{L} S+\left(J_{T}+m_{T} h_{T}^{2}+m_{T} l_{T} h_{T}\right) \ddot{T} L \\
& -\left(m_{L} g h_{L}+m_{T} g l_{L}+m_{T} g h_{T}\right) L S-\left(m_{T} g h_{T}\right) T L
\end{aligned}
$$

where $\ddot{L S}$ and $\ddot{T L}$ represent angular accelerations, $m_{L}$ and $m_{T}$ represent the leg and trunk segment masses, $l_{L}$ and $l_{T}$ represent the segment lengths, $h_{L}$ and $h_{T}$ represent the COM heights and $J_{L}$ and $J_{T}$ represent the moments of inertia around the segments' COM (details in Al Bakri, 2008). Note that angles in uppercase letters indicate physical angles, while the below given angles in lowercase letters are the sensory derived representations of these physical angles. 


\section{A.3. Disturbance estimations in the extended DEC model}

Ankle Module. The support surface tilt stimulus causes in the ankle joint the disturbances Foot-Space Tilt (FS), Gravitational Ankle Torque $\left(T_{A_{-} \text {grav }}\right)$, and External Ankle Torque $\left(T_{A_{-} \text {ext }}\right.$; compare Ankle Module in Fig. 5; further details in Mergner, 2010). In this module the three disturbances are estimated and compensated in the following form:

(i) Estimate of Foot-Space Tilt, $\widehat{F S}$. This estimate was obtained from the vestibular trunk-space angular velocity signal $\dot{t s}$, the proprioceptive trunk-leg angular velocity signal tl and the leg-foot velocity signal if as follows:

$$
\dot{\mathrm{fs}}=\dot{\mathrm{ts}}-\dot{\mathrm{tl}}-\dot{\mathrm{lf}} \text {. }
$$

The estimate $\widehat{F S}$ involves a velocity detection threshold, a gain factor and a mathematical integration, yielding the foot-space angular position signal $f s$. For disturbance compensation, $f_{s}$ was used to transform the leg-foot position signal If (from ankle joint proprioception $P R O P_{A}$ ) into a leg-space position signal $l s^{\prime \prime}$ Then a body-space position signal bs was computed as:

$$
b s=\frac{\left(h_{T} t s+l_{L} l s^{\prime \prime}\right) m_{T}+\left(h_{L} l s^{\prime \prime}\right) m_{L}}{h_{B} m_{B}}
$$

where $m_{B}$ represents the mass of the whole body and $h_{B}$ the whole body COM height. Small angular assumption allows approximating $h_{B}$ as a constant value.

(ii) Estimate of Gravitational Ankle Torque, $\widehat{T}_{A_{-} \text {grav }}$. For this estimate the vestibular trunk-space angular position signal $t s$ was combined with the proprioceptive trunk-leg position signal $t l$ to obtain a legspace position signal $l s=t s-t l$ and another version of the body-space position signal $b s^{\prime}$ (as in Eq. (4) but $l s^{\prime \prime}$ was substituted by $l s$ ). The $b s^{\prime}$ signal was processed using a position detection threshold and a gain factor. From this processed signal, the gravitational ankle torque was obtained in the form

$$
\widehat{T}_{\text {A_grav }}=m_{B} h_{B} g b s^{\prime} .
$$

A first order low-pass filtered feedback version of $b s^{\prime}$ with gain factor was added to $\widehat{T}_{A_{-} \text {grav }}$. This additional signal increased the compensation of the torque produced by body-space lean at low tilt frequencies such that the gain curves in the model simulations resembled those in the human data (compare 'tonic excursion limiter' in Schweigart \& Mergner, 2008). The effect of this additional signal is comparable to that achieved by the low-pass filtered torque feedback in the study of Peterka (2003) and the integrative part of the PID controller in Maurer et al. (2006). The motivation for the current solution was that the previous solutions produce a slow drift back, when a voluntary trunk lean was tested in the model. This kind of back drift occurs in the extended DEC model only with re-active body excursion during external disturbances and not with voluntary lean. The reason is that during voluntary lean, the sensory derived disturbance estimates are combined with predictive disturbance estimates (Mergner, 2010). The fusion of predictive and sensory signals in the estimate is thought to yield a close to ideal gain factor of unity and threshold values of 0 rad. As a consequence, there is no longer the effect of the low-pass filtered version of the bś signal during voluntary lean.

(iii) Estimate of External Ankle Torque, $\widehat{T}_{A_{-} e x t}$. Trunk rotational acceleration around the hip joints creates an inter-segmental coupling torque that evokes a counter rotation of the legs in the ankle joints. In the view of the DEC concept, the disturbance represents a 'push' against the body, comparable to a contact force (external torque) disturbance. The corresponding estimate was derived from a sensory signal of the active ankle torque $T_{a}$ and an internal estimate of the total ankle torque $T_{A} . T_{A}$ was obtained from body-space angular acceleration using the second derivative of the $b s$ signal from Eq. (4) in the form

$$
T_{A}=J_{B} \ddot{b s}
$$

where $J_{B}$ is the moment of inertia of the body around the ankle joint. The external ankle torque was obtained in the form

$$
\widehat{T}_{A \_ \text {ext }}=T_{A}-\widehat{T}_{A \_ \text {grav }}-T_{a}
$$


where $\widehat{T}_{A_{-} \text {grav }}$ was taken from Eq. (5). A more comprehensive description of $\widehat{T}_{A_{\_} \text {ext }}$ that includes inertial ankle torque arising with support surface translation and passive torque (intrinsic stiffness) is given in Mergner (2010). The estimate includes a gain factor and a low-pass filter (first-order filter with $0.8 \mathrm{~Hz}$ cutoff frequency) that eliminates effects of high-frequency components in the constituents of $\widehat{T}_{A_{-} e x t}$. In the current experimental conditions, the influence of $\widehat{T}_{A_{-} \text {ext }}$ on the simulation results was negligible. Therefore, the gain factor of $\widehat{T}_{A_{-} e x t}$ was not included in the parameter identification procedure and was assumed to be zero. Since the disturbance here occurs as a self-produced coupling force during hip rotation, we conceive that its estimate may be learned and predicted.

The ankle joint angle controller (box $\mathrm{C}_{\mathrm{A}}$ in Fig. 5) with proportional and derivative factors is used by both, the servo loop and the disturbance compensation loop. To generate the estimated compensation torques via the controller, a torque-to-angle conversion (box $B_{B}$ in Fig. 5) was applied to the estimated torques $\widehat{T}_{A_{-} \text {grav }}$ and $\widehat{T}_{A_{-} \text {ext }}$ by scaling them by $\frac{1}{m_{B} h_{B} g}$ using the small angle approximation (e.g., $\left.\arcsin \left(\widehat{T}_{A_{\text {grav }}} / m_{B} h_{B} g\right) \approx \widehat{T}_{A_{\text {grav }}} / m_{B} h_{B} g\right)$. Thus, first a body-space angle signal is here used to derive $\widehat{T}_{A_{-} \text {grav }}$. From this $\widehat{T}_{A_{-} \text {grav }}$ a corresponding body-space angle equivalent signal is derived, which via the controller generates the torque to counteract the gravitational disturbance acting on the body.

Hip Module. Human stabilization of the leg segment tends to be imperfect, leading to rotation and tangential acceleration at the level of the hip joint. Therefore, the Hip Module has to compensate for the disturbances Leg-Space Tilt (LS), Translational Acceleration ( $\dot{x}_{H}$; leads to inertial hip torque $T_{H \_i n}$ ), and Gravitational Hip Torque $\left(T_{H_{-} \text {grav }}\right)$, as shown in Fig. 5 . These three disturbances were estimated in the following form (parameter values are given in Table 1):

(i) Estimate of Leg-Space tilt, $\widehat{L S}$. This estimate was derived from the $\dot{t s}$ signal and the $\dot{t}$ signal in the form of

$$
\dot{l i s}=\dot{t s}-\dot{t} l
$$

Further processing comprised a velocity threshold, a gain factor and a mathematical integration to obtain the leg-space position signal $l s^{\prime}$. For compensation, which is in space coordinates, the trunkleg signal $t$ from hip joint proprioception $\left(P R O P_{H}\right)$ was either combined with $l s^{\prime}$ or with the up-channeled version of the leg-space signal $l s^{\prime \prime}$ as shown in Fig. 5.

(ii) Estimate of hip Translational Acceleration, $\widehat{\ddot{x}_{H}}$. In simplified form (see Mergner, 2010), this estimate can be derived from the vestibular head linear acceleration signal $\ddot{x}_{\text {Head }}$ and the trunk-space angular velocity signal $t$ s in the form

$$
\widehat{\ddot{x}_{H}}=\ddot{x}_{\text {Head }}-\frac{d(\dot{t} \dot{s})}{d t} l_{T}
$$

where $l_{T}$ represents the distance between the vestibular organ and the hip joint. The $\widehat{\ddot{x}_{H}}$ estimate was then used to obtain an estimate of inertial hip torque $\widehat{T}_{H_{-i n}}$ in the form

$$
\widehat{T}_{H \_i n}=\widehat{\ddot{x}_{H}} m_{T} h_{T} \text {. }
$$

The translational acceleration estimate was processed with a low-pass filter and a gain factor. This estimate contributes to the compensation of the coupling torque that arises from hip tangential acceleration during leg segment rotation (Hettich et al., 2011). Since the influence of this estimate on the simulation results was negligibly small, its gain factor was not included in the parameter identification procedure and was assumed to be zero. Similar to the external ankle torque disturbance, the translation acceleration disturbance occurs as a self-produced coupling force and may be learned and predicted.

(iii) Estimate of the Gravitational Hip Torque, $\widehat{T}_{H_{\text {grav }}}$. This estimate was obtained in analogy to Eq. (5) from ts in the form

$$
\widehat{T}_{H_{\text {grav }}}=m_{T} h_{T} \text { gts }
$$

and was processed by a detection threshold and gain factor. As in the Ankle Module, a first order lowpass filtered version of $t s$ with a gain factor was added to $\widehat{T}_{H_{-} \text {grav }}$.

Analogous to the Ankle Module, a torque-to-angle conversion was applied to the estimates $\widehat{T}_{H_{-} \text {grav }}$ and $\widehat{T}_{H_{-i n}}$ (box $B_{T}$ in Fig. 5). 


\section{References}

Al Bakri, M. (2008). Development of a mathematical model and simulation environment for the postural robot (PostuRob II). Available <http://www.posturob.uniklinik-freiburg.de>.

Alexandrov, A. V., Frolov, A. A., \& Massion, J. (2001a). Biomechanical analysis of movement strategies in human forward trunk bending. I. Modeling. Biological Cybernetics, 84, 425-434.

Alexandrov, A. V., Frolov, A. A., \& Massion, J. (2001b). Biomechanical analysis of movement strategies in human forward trunk bending II. Experimental study. Biological Cybernetics, 84, 435-443.

Alexandrov, A. V., Frolov, A. A., Horak, F. B., Carlson-Kuhta, P., \& Park, S. (2005). Feedback equilibrium control during human standing. Biological Cybernetics, 93, 309-322.

Amblard, B., Assaiante, C., Fabre, J.-C., Mouchnino, L., \& Massion, J. (1997). Voluntary head stabilization in space during oscillatory trunk movements in the frontal plane performed in weightlessness. Experimental Brain Research, 114, $214-225$.

Assaiante, C., \& Amblard, B. (1995). An ontogenetic model of sensorimotor organization of balance control in humans. Human Movement Science, 14, 13-43.

Bastian, A. J. (1997). Mechanisms of ataxia. Physical Therapy, 77, 672-675.

Bernstein, N. A. (1967). The co-ordination and regulation of movements. Oxford: Pergamon Press.

Berthoz, B., \& Pozzo, T. (1988). Intermittent head stabilization during postural and locomotory tasks in humans. In B. Amblard, A. Berthoz, \& F. Clarac (Eds.), Posture and gait: Development, adaptation and modulation (pp. 189-198). Amsterdam: Elsevier.

Boonstra, T. A., Schouten, A. C., \& van der Kooij, H. (2013). Identification of the contribution of the ankle and hip joints to multisegmental balance control. Journal of NeuroEngineering and Rehabilitation, 10, 23.

Bosco, G., \& Poppele, R. E. (1997). Representation of multiple kinematic parameters of the cat hindlimb in spinocerebellar activity. Journal of Neurophysiology, 78, 1421-1432.

Bouisset, S., \& Do, M. C. (2008). Posture, dynamic stability, and voluntary movement. Neurophysiologie Clinique, 38, $345-362$.

Bronstein, A. M. (1988). Evidence for a vestibular input contributing to dynamic head stabilization in man. Acta OtoLaryngologica, 105, 1-6.

Brooks, R., Breazeal, C., Marjanovic, M., Scassellati, B., \& Williamson, M. (1999). The cog project: Building a humanoid robot. In C. L. Nehaniv (Ed.). Computation for Metaphors, Analogy and Agents (Vol. 1562). New York: Springer.

Casabona, A., Valle, M. S., Bosco, G., \& Perciavalle, V. (2004). Cerebellar encoding of limb position. Cerebellum, 3, $172-177$.

Coulter, J. D., Mergner, T., \& Pompeiano, O. (1976). Effects of static tilt on cervical spinoreticular tract neurons. Journal of Neurophysiology, 39, 45-62.

Creath, R., Kiemel, T., Horak, F. B., Peterka, R. J., \& Jeka, J. J. (2005). A unified view of quiet and perturbed stance: Simultaneous co-existing excitable modes. Neuroscience Letters, 377, 75-80.

Creath, R., Kiemel, T., Horak, F. B., \& Jeka, J. (2008). The role of vestibular and somatosensory systems in intersegmental control of upright stance. Journal of Vestibular Research, 18, 39-49.

d'Avella, A., \& Pai, D. K. (2010). Modularity for sensorimotor control: Evidence and a new prediction. Journal of Motor Behavior, $42,361-369$.

Fregly, A. R. (1974). Vestibular ataxia and its measurement in man. In H. H. Kornhuber (Ed.), Handbook of sensory physiology. The vestibular system (pp. 321-361). New York: Springer.

Goodworth, A. D., \& Peterka, R. J. (2010). Influence of stance width on frontal plane postural dynamics and coordination in human balance control. Journal of Neurophysiology, 104, 1103-1118.

Goodworth, A. D. \& Peterka, R. J. (2012). Sensorimotor integration for multisegmental frontal plane balance control in humans. Journal of Neurophysiology, 107, 12-28.

Grossman, G. E., Leigh, R. J., Abel, L. A., Lanska, D. J., \& Thurston, S. E. (1988). Frequency and velocity of rotational head perturbation during locomotion. Experimental Brain Research, 70, 470-476.

Hauser, H., Neumann, G., Ijspeert, A. J., \& Maass, W. (2011). Biologically inspired kinematic synergies enable linear balance control of a humanoid robot. Biological Cybernetics, 104, 235-249.

Hettich, G., Fennell, L., \& Mergner, T. (2011). Double inverted pendulum model of reactive human stance control. Multibody dynamics conference 2011. Available <http://www.posturob.uniklinik-freiburg.de>.

Hettich, G., Lippi, V., \& Mergner, T. (2013). Human-like sensor fusion mechanisms in a postural control robot. In: A. E. Londral, P. Encarnação, \& J. L. Pons (Eds.), Proceedings of the international congress on neurotechnology, electronics and informatics (pp. 152-160), 18-20 September, 2013. Available <http://www.posturob.uniklinik-freiburg.de>.

Horak, F. B., \& Nashner, L. M. (1986). Central programming of postural movements: Adaptation to altered support-surface configurations. Journal of Neurophysiology, 55, 1369-1381.

Horak, F. B., \& Macpherson, J. M. (1996). Postural orientation and equilibrium. In L. Rowell \& J. Shepherd (Eds.). Handbook of Physiology (Vol. 1, pp. 225-292). New York: Oxford University Press.

Ivanenko, Y. P., Cappellini, G., Dominici, N., Poppele, R. E., \& Lacquaniti, F. (2005). Coordination of locomotion with voluntary movements in humans. Journal of Neuroscience, 25, 7238-7253.

Kiemel, T., Elahi, A. J., \& Jeka, J. J. (2008). Identification of the plant for upright stance in humans: Multiple movement patterns from a single neural strategy. Journal of Neurophysiology, 100, 3394-3406.

Kuo, A. D. (2005). An optimal state estimation model of sensory integration in human postural balance. Journal of Neural Engineering, 2, 235-249.

Kutch, J. J., \& Valero-Cuevas, F. J. (2012). Challenges and new approaches to proving the existence of muscle synergies of neural origin. PLoS Computational Biology, 8, e1002434.

Lippi, V., Mergner, T. \& Hettich, G. (2013). A Bio-inspired Modular System for Humanoid Posture Control. In: Ugur, E. Oztop, E. Morimoto, J., Ishii, S. (Eds.), Proceedings of IROS 2013 workshop on neuroscience and robotics, Towards a robot-enabled, neuroscience-guided healthy society, November 3rd, (pp. 16-21). Tokyo, Japan.

Lockhart, D. B., \& Ting, L. H. (2007). Optimal sensorimotor transformations for balance. Nature Neuroscience, 10, 1329-1336.

Massion, J. (1992). Movement, posture and equilibrium: Interaction and coordination. Progress in Neurobiology, 38, 35-56.

Maurer, C., \& Peterka, R. J. (2005). A new interpretation of spontaneous sway measures based on a simple model of human postural control. Journal of Neurophysiology, 93, 189-200. 
Maurer, C., Mergner, T., \& Peterka, R. J. (2006). Multisensory control of human upright stance. Experimental Brain Research, 171, $231-250$.

McCollum, G., \& Leen, T. K. (1989). Form and exploration of mechanical stability limits in erect stance. Journal of Motor Behavior, $21,225-244$.

McCollum, G., Horak, F. B., \& Nashner, L. M. (1984). Parsimony in neural calculations for postural movements. In J. Bloedel, J. Dichgans, \& W. Precht (Eds.), Cerebellar functions (pp. 52-66). Berlin: Springer.

Mergner, T., Nardi, G. L., Becker, W., \& Deecke, L. (1983). The role of canal-neck interaction for the perception of horizontal trunk and head rotation. Experimental Brain Research, 49, 198-208.

Mergner, T., Siebold, C., Schweigart, G., \& Becker, W. (1991). Human perception of horizontal trunk and head rotation in space during vestibular and neck stimulation. Experimental Brain Research, 85, 389-404.

Mergner, T., Huber, W., \& Becker, W. (1997). Vestibular-neck interaction and transformations of sensory coordinates. Journal of Vestibular Research, 7, 119-135.

Mergner, T., \& Rosemeier, T. (1998). Interaction of vestibular, somatosensory and visual signals for posture control and motion perception under terrestrial and microgravity conditions. Brain Research Reviews, 28, 118-135.

Mergner, T., Maurer, C., \& Peterka, R. J. (2003). A multisensory posture control model of human upright stance. Progress in Brain Research, 142, 189-201.

Mergner, T. (2004). Meta level concept versus classic reflex concept for the control of posture and movement. Archives Italiennes De Biologie, 142, 175-198.

Mergner, T., Huethe, F., Maurer, C., \& Ament, C. (2006). Human equilibrium control principles implemented into a biped robot. In: T. Zielinska \& C. Zielinski (Eds.), Robot design, dynamics, and control (pp. 271-279) CISM courses and lectures.

Mergner, T., Schweigart, G., \& Fennell, L. (2009). Vestibular humanoid postural control. Journal of Physiology-Paris, 103, $178-194$.

Mergner, T., Schweigart, G., Fennell, L., \& Maurer, C. (2009). Posture control in vestibular loss patients. Annals of the New York Academy of Sciences, 1164, 206-215.

Mergner, T. (2010). A neurological view on reactive human stance control. Annual Reviews in Control, 34, 177-198.

Nashner, L. M., \& Berthoz, A. (1978). Visual contribution to rapid responses during postural control. Brain Research, 150, 403-407.

Nashner, L., \& McCollum, G. (1985). The organization of human postural movements: A formal basis and experimental synthesis. The Behavioral and Brain Sciences, 8, 135-172.

Otnes, R. K., \& Enochson, L. D. (1972). Digital Time Series Analysis. New York: Wiley.

Pai, Y. C., \& Patton, J. L. (1997). Center of mass velocity-position predictions for balance control. Journal of Biomechanics, 30 347-354.

Park, S., Horak, F. B., \& Kuo, A. D. (2004). Postural feedback responses scale with biomechanical constraints in human standing. Experimental Brain Research, 154, 417-427.

Peterka, R. J., \& Benolken, M. S. (1995). Role of somatosensory and vestibular cues in attenuating visually induced human postural sway. Experimental Brain Research, 105, 101-110.

Peterka, R. J. (2002). Sensorimotor integration in human postural control. Journal of Neurophysiology, 88, 1097-1118.

Peterka, R. J. (2003). Simplifying the complexities of maintaining balance. IEEE Engineering in Medicine and Biology Magazine, 22, 63-68.

Pozzo, T., Berthoz, A., Lefort, L., \& Vitte, E. (1990). Head stabilization during various locomotor tasks in humans. II. Patients with bilateral peripheral vestibular deficits. Experimental Brain Research, 85, 208-217.

Rugy, A., Loeb, G. E., \& Carroll, T. J. (2012). Muscle coordination is habitual rather than optimal. Journal of Neuroscience, 32, 7384-7391.

Runge, C. F., Shupert, C. L., Horak, F. B., \& Zajac, F. E. (1999). Ankle and hip postural strategies defined by joint torques. Gait and Posture, 10, 161-170.

Schweigart, G., \& Mergner, T. (2008). Human stance control beyond steady state response and inverted pendulum simplification. Experimental Brain Research, 185, 635-653.

Suzuki, Y., Nomura, T., \& Morraso, P. (2011). Stability of a double inverted pendulum model during human quiet stance with continuous delay feedback control. In: Proceedings of the annual international conference of the IEEE engineering in medicine and biology society, EMBS, art. no. 6091747, pp. 7450-7453.

Suzuki, Y., Nomura, T., Casadio, M., \& Morasso, P. (2012). Intermittent control with ankle, hip, and mixed strategies during quiet standing A theoretical proposal based on a double inverted pendulum model. Journal of Theoretical Biology, 310, 55-79.

Ting, L. H. (2007). Dimensional reduction in sensorimotor systems: A framework for understanding muscle coordination of posture. Progress in Brain Research, 165, 301-325.

Tresch, M. C., Saltiel, P., \& Bizzi, E. (1999). The construction of movement by the spinal cord. Nature Neuroscience, 2, $162-167$.

van der Kooij, H., Jacobs, R., Koopman, B., \& Grootenboer, H. (1999). A multisensory integration model of human stance control. Biological Cybernetics, 80, 299-308.

van der Kooij, H., van Asseldonk, E., \& van der Helm, F. C. T. (2005). Comparison of different methods to identify and quantify balance control. Journal of Neuroscience Methods, 145, 175-203.

van der Kooij, H., \& Peterka, R. J. (2011). Non-linear stimulus-response behavior of the human stance control system is predicted by optimization of a system with sensory and motor noise. Journal of Computational Neuroscience, 30, 759-778.

van Soest, A. J., Haenen, W. P., \& Rozendaal, L. A. (2003). Stability of bipedal stance: The contribution of cocontraction and spindle feedback. Biological Cybernitcs, 88, 293-301.

Vernazza-Martin, S., Martin, N., Le Pellec-Muller, A., Tricon, V., \& Massion, J. (2006). Kinematic synergy adaptation to an unstable support surface and equilibrium maintenance during forward trunk movement. Experimental Brain Research, 173, 62-78.

Winter, D. A. (1990). Biomechanics and motor control of human movement (2nd ed.). New York: Wiley-Interscience.

Winter, D. A., Patla, A. E., Prince, F., Ishac, M., \& Gielo-Perczak, K. (1998). Stiffness control of balance in quiet standing. Journal of Neurophysiology, 80, 1211-1221.

Zajac, F. E., \& Gordon, M. E. (1989). Determining muscle's force and action in multi- articular movement. Exercise and Sport Science Reviews, 17, 187-230. 